\title{
T-RaMiSu: the Two-meter Radio Mini Survey
}

\section{The Boötes Field ${ }^{\star}$}

\author{
W. L. Williams ${ }^{1,2}$, H. T. Intema ${ }^{3, \star \star}$, and H. J. A. Röttgering ${ }^{1}$ \\ ${ }^{1}$ Leiden Observatory, Leiden University, PO Box 9513, 2300 RA Leiden, The Netherlands \\ e-mail: wwilliams@strw.leidenuniv.nl \\ 2 Netherlands Institute for Radio AStronomy (ASTRON), PO Box 2, 7990 AA Dwingeloo, The Netherlands \\ ${ }^{3}$ National Radio Astronomy Observatory, 520 Edgemont Road, Charlottesville, VA 22903-2475, USA
}

Received 15 August 2012 / Accepted 31 October 2012

\section{ABSTRACT}

\begin{abstract}
We present wide area, deep, high-resolution $153 \mathrm{MHz}$ GMRT observations of the NOAO Boötes field, adding to the extensive, multiwavelength data of this region. The observations, data reduction, and catalogue construction and description are described here. The seven pointings produced a final mosaic covering 30 square degrees with a resolution of $25^{\prime \prime}$. The rms noise is $2 \mathrm{mJy}^{-1}$ beam ${ }^{-1}$ in the centre of the image, rising to $4-5 \mathrm{mJy}_{\text {beam }}{ }^{-1}$ on the edges, with an average of $3 \mathrm{mJy}_{\text {beam }}{ }^{-1}$. Seventy-five per cent of the area has an rms $<4$ mJy beam $^{-1}$. The extracted source catalogue contains 1289 sources detected at $5 \sigma$, of which 453 are resolved. We estimate the catalogue to be 92 per cent reliable and 95 per cent complete at an integrated flux density limit of 14 mJy. The flux densities and astrometry have been corrected for systematic errors. We calculate the differential source counts, which are in good agreement with those in the literature and provide an important step forward in quantifying the source counts at these low frequencies and low flux densities. The GMRT $153 \mathrm{MHz}$ sources have been matched to the $1.4 \mathrm{GHz}$ NVSS and $327 \mathrm{MHz}$ WENSS catalogues and spectral indices were derived.
\end{abstract}

Key words. techniques: interferometric - surveys - galaxies: active - radio continuum: galaxies

\section{Introduction}

Deep low-frequency radio surveys provide unique data which will help resolve many questions related to the formation and evolution of massive galaxies, quasars and galaxy clusters. Until now, such surveys have largely been limited by the corrupting influence of the ionosphere on the visibility data, but new techniques allow for the correction for these effects (e.g. Cotton et al. 2004; Intema et al. 2009). Recently deep $\left(0.7-2 \mathrm{mJy}\right.$ beam $\left.^{-1}\right)$ images have been made, in particular with the Giant Metrewave Radio Telescope (GMRT, e.g. Ananthakrishnan 2005) at $153 \mathrm{MHz}$ (e.g. Ishwara-Chandra \& Marathe 2007; Sirothia et al. 2009; Ishwara-Chandra et al. 2010). These observations can be used to study:

Luminous radio sources at $z>4$ - High redshift radio galaxies (HzRGs, e.g. Miley \& De Breuck 2008) provide a unique way to study the evolution of the most massive galaxies in the Universe. One of the most efficient ways of identifying these sources is to search for ultra-steep spectrum (USS) radio sources with $\alpha \lesssim-1, S_{v} \propto v^{\alpha}$ (Röttgering et al. 1997; De Breuck et al. 2002). Low frequency observations provide an easy way of identifying USS sources and extending these observations to lower flux density limits increases the distance to which these HzRGs can be identified. Surveying larger areas increases the probability of locating these rare sources.

\footnotetext{
* Table A.1 (Catalogue) is only available in electronic form at the CDS via anonymous ftp to cdsarc.u-strasbg. fr (130.79.128.5) or via

http://cdsarc.u-strasbg.fr/viz-bin/qcat?]/A+A/549/A55

$\star \star$ Jansky Fellow of the National Radio Astronomy Observatory.
}

Distant starburst galaxies - The local radio-IR correlation for star forming galaxies is very tight, and seems to hold at high redshift (Kovács et al. 2006). However, the physical processes involved are poorly understood and only models that carefully fine-tune the time scales for the heating of the dust, the formation of supernovae, and the acceleration, diffusion and decay of the relativistic electrons can reproduce the correlation. The lowfrequency spectral shape of galaxies reveals information about the amount of free-free absorption and relating this to the dust content, size, mass, total amount of star formation and environment of the galaxies will further constrain the radio-IR models. To date, however, few galaxies have been well studied at low frequencies and those that have, show a diverse range of spectral shapes (e.g. Clemens et al. 2010).

Faint peaked spectrum sources - Young radio-loud active galactic nuclei (AGN) are ideal objects to study the onset and early evolution of classical double radio sources. They usually have synchrotron self-absorbed spectra and compact radio morphologies. Relative number statistics have indicated that these radio sources must be significantly more powerful at young ages, which may be preceded by a period of luminosity increase (e.g. Snellen et al. 2003). Multi-epoch VLBI observations of individual Gigahertz Peaked Spectrum and Compact Symmetric Objects indicate dynamical ages in the range of a few hundred to a few thousand years (e.g. Polatidis \& Conway 2003). Since the peak of these sources shifts to lower observed frequencies at higher redshift, low frequency observations, combined with multi-wavelength data, can identify these faint peakers and establish whether they are less luminous or at very high redshift and have different host properties (masses, starformation rates). 
The accretion modes of radio sources - Radio galaxies and radio loud quasars have been studied extensively in order to reveal the details of the relationship between AGN and their host galaxies, in particular how their interaction affects their evolution. The expanding jets of radio-loud AGN provide a mechanism for the transfer of energy to the intracluster medium and prevent the catastrophic cooling and formation of too-massive elliptical galaxies (Fabian et al. 2006; Best et al. 2006, 2007; Croton et al. 2006; Bower et al. 2006), but the accretion and feedback processes and how they evolve over cosmic time are not fully understood. It is known that the fraction of massive galaxies which are radio-loud at $z \sim 0.5$ is about the same as observed locally ( $z \sim 0.1$, Best et al. 2005), while for less massive galaxies $\left(<10^{10.5} M_{\odot}\right)$, it is an order of magnitude larger. Studies of these AGN show two different types: a "hot" mode where radiatively inefficient accretion occurs from hot halo gas onto massive galaxies, and a "cold" mode where cold gas from major mergers drives high accretion rates. The strong evolution in the radio luminosity function is thus a result of less massive galaxies experiencing more mergers and being more active at high $z$. A full understanding of the different AGN populations, their distribution in luminosity and host galaxy properties, and particularly their cosmic evolution, is important for AGN and galaxy evolutionary models. Differences in their host galaxy populations will provide insight into the triggering mechanisms for radio activity as well as the effect of radio feedback.

In this paper we present wide, deep, high-resolution observations of the NOAO Boötes extra-galactic field at $153 \mathrm{MHz}$ taken with the GMRT. An initial, very deep, $\sim 1 \mathrm{mJy}_{\text {beam }}{ }^{-1} \mathrm{rms}$, $153 \mathrm{MHz}$ GMRT map of this field was presented by Intema et al. (2011). Here we present additional pointings around this map effectively tripling the size of the surveyed area at a slightly higher noise level. The Boötes field is part of the NOAO Deep Wide Field Survey (NDWFS; Jannuzi et al. 1999) and covers $\sim 9 \mathrm{deg}^{2}$ in the optical and near infra-red $B_{W}, R, I$ and $K$ bands. There is a wealth of additional complementary data available for this field, including X-ray (Murray et al. 2005; Kenter et al. 2005), UV (GALEX; Martin et al. 2003), and mid infrared (Eisenhardt et al. 2004; Martin et al. 2003). The region has also been surveyed at radio wavelengths with the WSRT at $1.4 \mathrm{GHz}$ (de Vries et al. 2002), the VLA at $1.4 \mathrm{GHz}$ (Higdon et al. 2005) and $325 \mathrm{MHz}$ (Croft et al. 2008). Recently, the AGN and Galaxy Evolution Survey (AGES) has provided redshifts for 23745 galaxies and AGN across $7.7 \mathrm{deg}^{2}$ of the Boötes field (Kochanek et al. 2012). This unique rich multiwavelength dataset, combined with the new low frequency radio data presented here, will be valuable in improving our understanding of the above-mentioned key topics in astrophysics.

The observations presented here are the first part of the Two-meter Radio Mini Survey (T-RaMiSu), consisting of two $153 \mathrm{MHz}$ mosaics of similar area and depth. The second mosaic, centered on the galaxy cluster Abell 2256, will be presented by Intema et al. (in prep.).

This paper is structured as follows. In Sect. 2 we describe the GMRT observations of the extended region around the NOAO Boötes field. We describe the techniques employed to achieve the deepest possible images. Our data reduction relies on the ionospheric calibration with the SPAM package (Intema et al. 2009). In Sect. 3 we describe the source detection method and the compilation of a source catalogue. This section also includes a discussion of the completeness and reliability of the catalogue and an analysis of the quality of the catalogue. The source counts and spectral index distributions are presented in Sect. 4. Finally, Sect. 5 summarises and concludes this work.
Table 1. GMRT observation parameters for the Boötes field.

\begin{tabular}{lcc}
\hline \hline Parameter & Central & Flanking \\
\hline Observation dates & 3 June 2005 & 3-6 June 2006 \\
Pointings & Boötes & Boötes A-F \\
Primary calibrator & 3C 48 & 3C 48 \\
Total time on calibrator & $20 \mathrm{~min}$ & $51 \mathrm{~min}$ \\
Secondary calibrator & $3 \mathrm{C} 286$ & $3 \mathrm{C} 286$ \\
Total time on calibrator & $9 \times 10 \mathrm{~min}$ & $10 \times 4.5 \mathrm{~min}$ (per day) \\
Cadence & $50 \mathrm{~min}$ & $30 \mathrm{~min}$ \\
Total time on target & $359 \mathrm{~min}$ & $205 \mathrm{~min}$ (per pointing) \\
\hline Integration time & & $16.9 \mathrm{~s}$ \\
Polarisations & $\mathrm{RR}, \mathrm{LL}$ \\
Channels & 128 \\
Channel width & & $62.5 \mathrm{kHz}$ \\
Total bandwidth & & $8.0 \mathrm{MHz}$ \\
Central frequency & & $153 \mathrm{MHz}$ \\
\hline
\end{tabular}

Table 2. Pointing centres of the Boötes central and flanking fields.

\begin{tabular}{lcc}
\hline \hline Field & $\begin{array}{c}\text { RA } \\
(\mathrm{J} 2000)\end{array}$ & $\begin{array}{c}\text { Dec } \\
(\mathrm{J} 2000)\end{array}$ \\
\hline Boötes & $14: 32: 05.75$ & $+34: 16: 47.5$ \\
Boötes A & $14: 32: 05.75$ & $+36: 06: 47.5$ \\
Boötes B & $14: 24: 19.53$ & $+35: 10: 52.5$ \\
Boötes C & $14: 24: 29.58$ & $+33: 20: 54.5$ \\
Boötes D & $14: 32: 05.75$ & $+32: 26: 47.5$ \\
Boötes E & $14: 39: 41.92$ & $+33: 20: 54.5$ \\
Boötes F & $14: 39: 51.97$ & $+35: 10: 52.5$ \\
\hline
\end{tabular}

\section{Observations and data reduction}

\subsection{Observations}

The central Boötes field was previously observed with the GMRT from 3-4 June 2005 (Intema et al. 2011). We use the data from a single day of this observing run, combined with new observations of six flanking fields taken during 3-6 June 2006 with the GMRT at $153 \mathrm{MHz}$. Data from the first day only of the first observing run, 3 June, was used as the RFI situation was marginally better on this day and the length of a single day's observation, $359 \mathrm{~min}$, compares well with that of the new observations of the flanking fields, $205 \mathrm{~min}$, which leads to a more uniform mosaic. Table 1 lists the observational parameters used, highlighting any differences between the two sets of observations. The flanking fields are arranged on a hexagonal grid with a radius of $110^{\prime}$ just beyond the half power point of the primary beam of the GMRT at $153 \mathrm{MHz}\left(\theta_{\text {FWHM }} \sim 3^{\circ}\right)$; Table 2 gives the central coordinates of each pointing. Typically 26-27 of the 30 antennas were available during each observing run. 3C 48 and 3C 286 were observed as phase, bandpass and flux density calibrators. For each of the four days, the target fields were observed in sets of $\sim 4.5 \mathrm{~min}$ each, followed by a calibrator observation (3C 286) of $\sim 4.5 \mathrm{~min}$. 3C 48 was observed at the beginning or end of each day for $\sim 20-30 \mathrm{~min}$. The frequent ( $\sim 30$ min interval) calibrator observations of 3C 286 provide a means to track changes in the GMRT system, RFI and ionospheric conditions, and flux density scale. The short target field observations spread over each night of observing provides fairly uniform $u v$-coverage.

\subsection{Data reduction}

The data for the central pointing was re-reduced in the same manner as the new flanking fields in order to allow for consistent integration into a single mosaic. The data reduction consisted 
Table 3. Final CLEANing (top) and SPAM (bottom) parameters for individual Boötes fields.

\begin{tabular}{lc}
\hline \hline Parameter & Value $^{-}$ \\
\hline Widefield imaging & $\begin{array}{c}\text { polyhedron facet-based }^{a} \\
\text { multi-frequency synthesis }\end{array}$ \\
Deconvolution & Cotton-Schwab CLEAN $^{c}$ \\
field size & $4^{\circ}$ \\
facets & 85 \\
facet size & $32.4^{\prime}$ \\
facet separation & $26.4^{\prime}$ \\
Weighting & Robust $^{e}-0.5^{d}$ \\
UVBXFN, UVBOX & 4,1 \\
CLEAN box threshold & $5 \sigma$ \\
CLEAN depth & $3 \sigma$ \\
Pixel size & $3.8^{\prime \prime}$ \\
Restoring beam & $25^{\prime \prime}$ circular $^{d}$ \\
\hline SPAM calibration cycles & 3 \\
Peeled sources & $20^{f}$ \\
Layer heights (weights) & $250 \mathrm{~km}(0.5)$ \\
Turbulence parameter $\gamma$ & $350 \mathrm{~km}^{g}(0.5)$ \\
Model parameters & $5 / 3^{g}$ \\
Reference catalogue & $\leq 20$ \\
\hline
\end{tabular}

Notes. ${ }^{(a)}$ Perley (1989); Cornwell \& Perley (1992). ${ }^{(b)}$ Conway et al. (1990). (c) Schwab (1984); Cotton (1999); Cornwell et al. (1999). ${ }^{(d)}$ Final imaging parameters. ${ }^{(e)}$ Briggs (1995). ${ }^{(f)} 14$ for field F. ${ }^{(g)}$ Pure Kolmogorov turbulence. ${ }^{\left({ }^{(}\right)}$Condon et al. $(1994,1998)$.

of two stages: "traditional calibration" followed by directionaldependent ionospheric phase calibration, both of which were implemented in Python using the ParselTongue (Kettenis et al. 2006) interface to the Astronomical Image Processing System package (AIPS; Greisen 1998). Ionospheric calibration was done with the "Source Peeling and Atmospheric Modelling" ParselTongue-based Python module (SPAM; Intema et al. 2009).

The data for each day were calibrated separately. The flux density scale was set and initial amplitude, phase and bandpass calibration were done using $3 \mathrm{C} 48.3 \mathrm{C} 48$ is brighter than 3C 286 and provides a better determination of the bandpass. To reduce the data volume, the LL and RR polarisations were combined as Stokes $I$ and every 5 channels were combined to form 18 channels of width $0.3125 \mathrm{MHz}$ yielding an effective bandwidth of $5.625 \mathrm{MHz}$. After this calibration, the $u v$-data from all four days for each target, were combined.

Initial imaging of each target field was done after a phase-only calibration against a model field constructed from NVSS sources within each field. Table 3 lists the important imaging parameters. The calibration was then improved by several rounds of phase-only self-calibration followed by one round of amplitude and phase self-calibration where gain solutions were determined on a longer time-scale than the phase-only solutions. Excessive visibilities were determined from the modelsubtracted data and were removed. Additional automated removal of bad data causing ripples in the image plane was done by Fourier transforming the model-subtracted images and identifying and removing extraneous peaks in the $u v$-plane. Further, persistent RFI was flagged and low level RFI modelled and subtracted using the LowFRFI ${ }^{1}$ routine in ObitTalk (Cotton 2008). After self-calibration the rms noise in the inner half of the primary beam area was $2.5 \mathrm{mJy}^{\text {beam }}{ }^{-1}$ in the central field and $3.5-5 \mathrm{mJy}$ beam $^{-1}$ in the flanking fields, with the local noise

\footnotetext{
1 Obit Development Memo Series \# 16 see

http://www.cv.nrao.edu/ bcotton/Obit.html
}

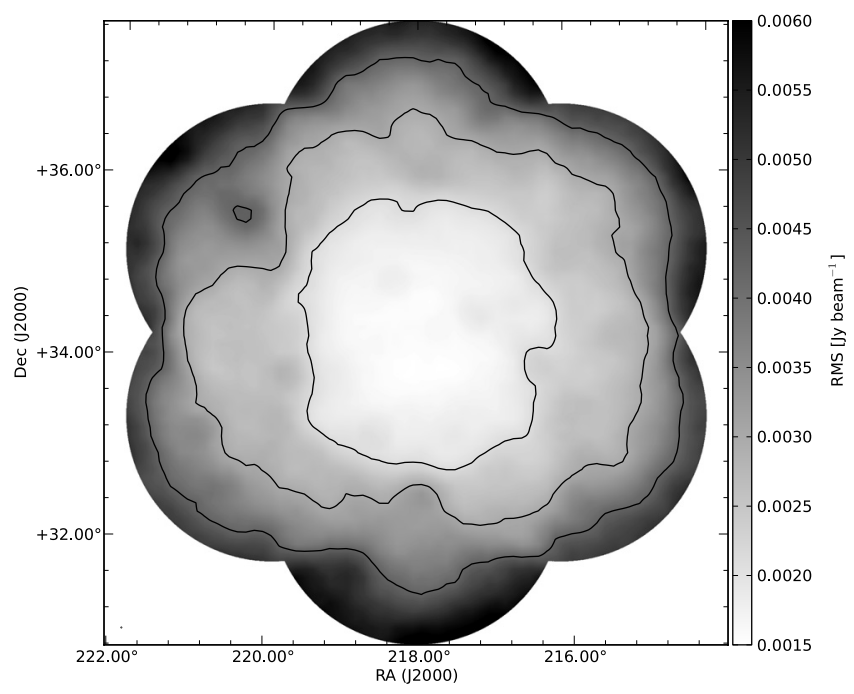

Fig. 1. Greyscale map showing the local rms noise measured in the mosaic image. The greyscale shows the rms noise from $0.5 \sigma_{\text {avg }}$ to $2 \sigma_{\text {avg }}$, where $\sigma_{\mathrm{avg}}=3.0 \mathrm{mJy} \mathrm{beam}^{-1}$ is the approximate $\mathrm{rms}$ in the mosaic centre. The contours are plotted at $[1 / \sqrt{2}, 1, \sqrt{2}] \times \sigma_{\text {avg. }}$. Peaks in the local noise coincide with the locations of bright sources.

increasing 2-3 times near the brightest sources. Note, the presence of extremely bright sources with peak flux densities of the order of 5-8 $\mathrm{Jy} \mathrm{beam}^{-1}$ prior to primary beam correction in flanking fields D through $F$ resulted in the slightly higher overall noise in these fields.

Significant artefacts, however, remained in all fields near bright sources. To reduce these we applied the SPAM algorithm on the self-calibrated data. The SPAM parameters are listed in the bottom part of Table 3 which include the number of ionospheric layers modelled and their heights and relative weights, the slope of the assumed power law function of phase structure resulting from turbulence $(\gamma)$ and the number of free parameters in the fit; see Intema et al. (2009) for a more detailed description of the meaning of these parameters. Three iterations of peeling were done: in the first we only applied the peeling solutions to the peeled sources and in the final two we fitted an ionospheric phase screen to the peeling solutions. Up to 20 sources with flux densities above $0.4 \mathrm{Jy}$ (not corrected for primary beam effects) were peeled in the final stage in each field. The screen was made up of two equally-weighted turbulent layers at 250 and $350 \mathrm{~km}$. SPAM also allowed for the determination of and correction for antenna-based phase discontinuities.

To have homogeneous point spread functions in all pointings, final images were made with a circular restoring beam of radius $25^{\prime \prime}$ and a pixel size of $3.8^{\prime \prime}$. The flux density scales of the flanking fields were scaled up by 30 per cent based on information from 3C 286 (discussed in Sect. 3.4). In the final individual field images, the rms noise in the central half of the primary beam area before primary beam correction was $1.8 \mathrm{mJy}^{\text {beam }}{ }^{-1}$ in the central field and $2.5-2.7 \mathrm{mJy} \mathrm{beam}^{-1}$ respectively in the flanking fields. This is $3-5$ times the theoretical noise, similar to the factor above thermal noise obtained by the deeper single pointing of Intema et al. (2011). The seven pointings were each corrected for the primary beam of the GMRT up to a radius of $1.6^{\circ}$, where the primary beam correction factor drops to 40 per cent of its central value, and were then mosaicked together by weighting the final image by the inverse of the square of the rms noise of each individual pointing. Figure 1 illustrates the variation in rms noise across the mosaic which is shown in entirety in Fig. 2. The noise level is smooth and 


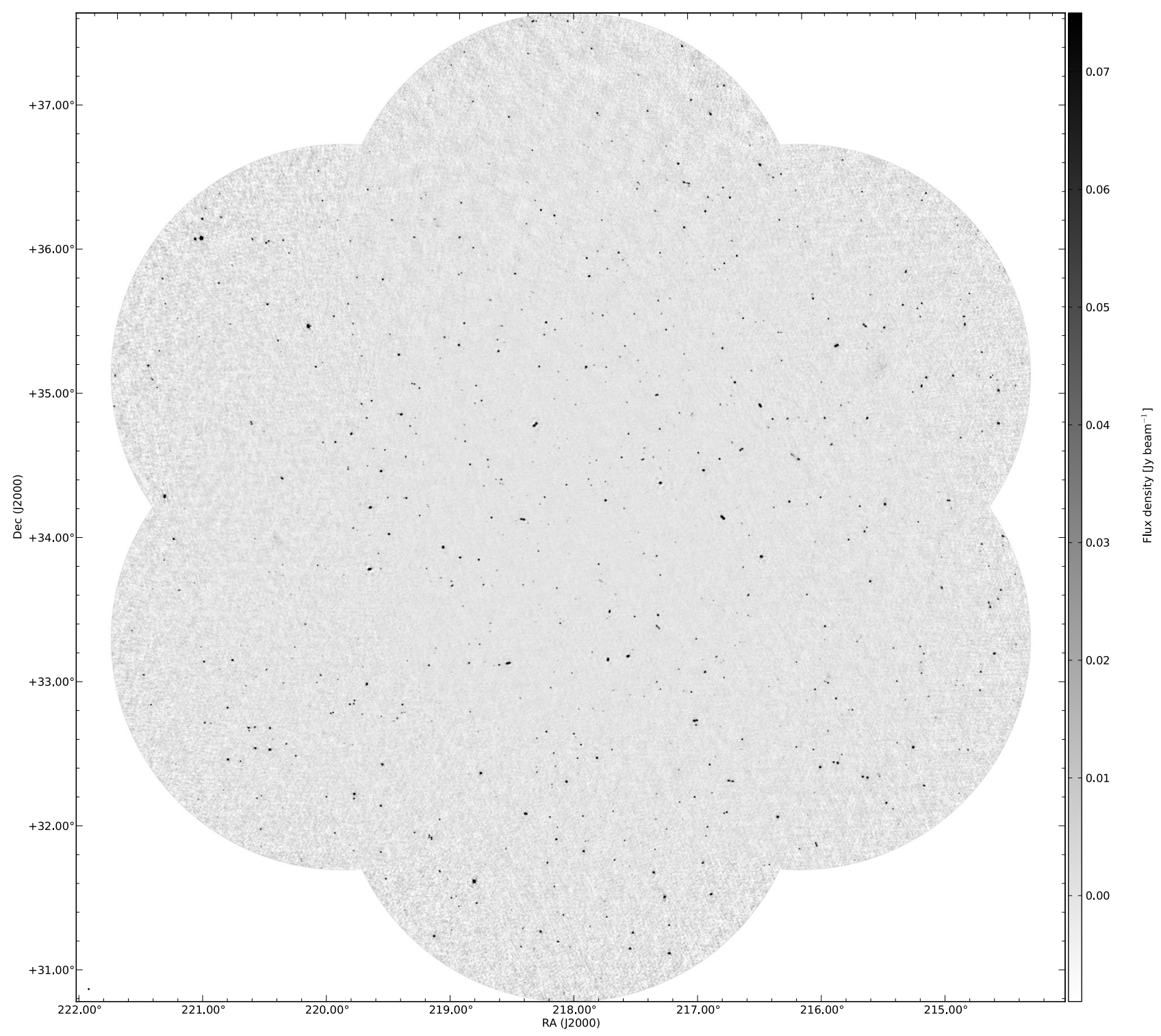

Fig. 2. Greyscale map showing the entire mosaic. The image covers 30 square degrees. The greyscale shows the flux density from $-3 \sigma_{\text {avg }}$ to $25 \sigma_{\text {avg }}$ where $\sigma_{\mathrm{avg}}=3.0 \mathrm{mJy}^{-1}$ beam $^{-1}$ is the average rms across the entire mosaic.

around $2 \mathrm{mJy}_{\text {beam }}^{-1}$ across the interior of the map, and increases towards the edges to about $4-5 \mathrm{mJy}_{\text {beam }}{ }^{-1}$. The average noise in the final mosaic is $3.0 \mathrm{mJy}^{-}$beam $^{-1}$, with 49 per cent under $3 \mathrm{mJy}^{\text {beam }}{ }^{-1}$ and 74 per cent under $4 \mathrm{mJy}^{\text {beam }}{ }^{-1}$. A small portion of the mosaic covering the inner square degree is shown in Fig. 3 to illustrate the resolution and quality of the map. There remain some phase artefacts visible around the brightest sources, which have not been entirely removed during peeling. It is possible that some artefacts are caused by elevation-dependent pointing errors, since each pointing was observed in a series of scans with varying elevations (Tasse et al. 2007; Mohan et al. 2001; Chandra et al. 2004).

\section{Source detection and characterisation}

\subsection{Detection}

We used the PyBDSM package ${ }^{2}$ to detect and characterise sources in the mosaic image. PyBDSM identifies islands of

2 http://home.strw.leidenuniv.nl/ mohan/anaamika contiguous emission by identifying all pixels greater than the pixel threshold and adding each of these pixels to an island of contiguous pixels exceeding the island threshold. Each island is fit with one or more Gaussians which are subsequently grouped into sources. Sources are classified as "S" for single sources, " $\mathrm{M}$ " for multiple-Gaussian sources and " $\mathrm{C}$ " for components of a multi-source island. From the fitted parameters the deconvolved sizes are computed assuming the theoretical beam. Errors on the fitted parameters are computed following Condon (1997). Prior to source detection the local background rms is determined by measuring the pixel statistics within a sliding box. For determining the rms background in our map we used a box size of 100 pixels to capture the variation in local noise around the brightest sources. We used a pixel threshold of $5 \sigma_{\mathrm{L}}$ and an island threshold of $3 \sigma_{\mathrm{L}}$. In generating a source list we allowed all Gaussians in each island to be grouped into a single source. PyBDSM detected 1296 sources from 1578 Gaussians fitted to 1301 islands, of which 1073 were single-component "S" sources. Based on visual inspection a small number of sources were removed as they were false, or bad, detections on the edge of the image. 
W. L. Williams et al.: T-RaMiSu: The Two-meter Radio Mini Survey. I.

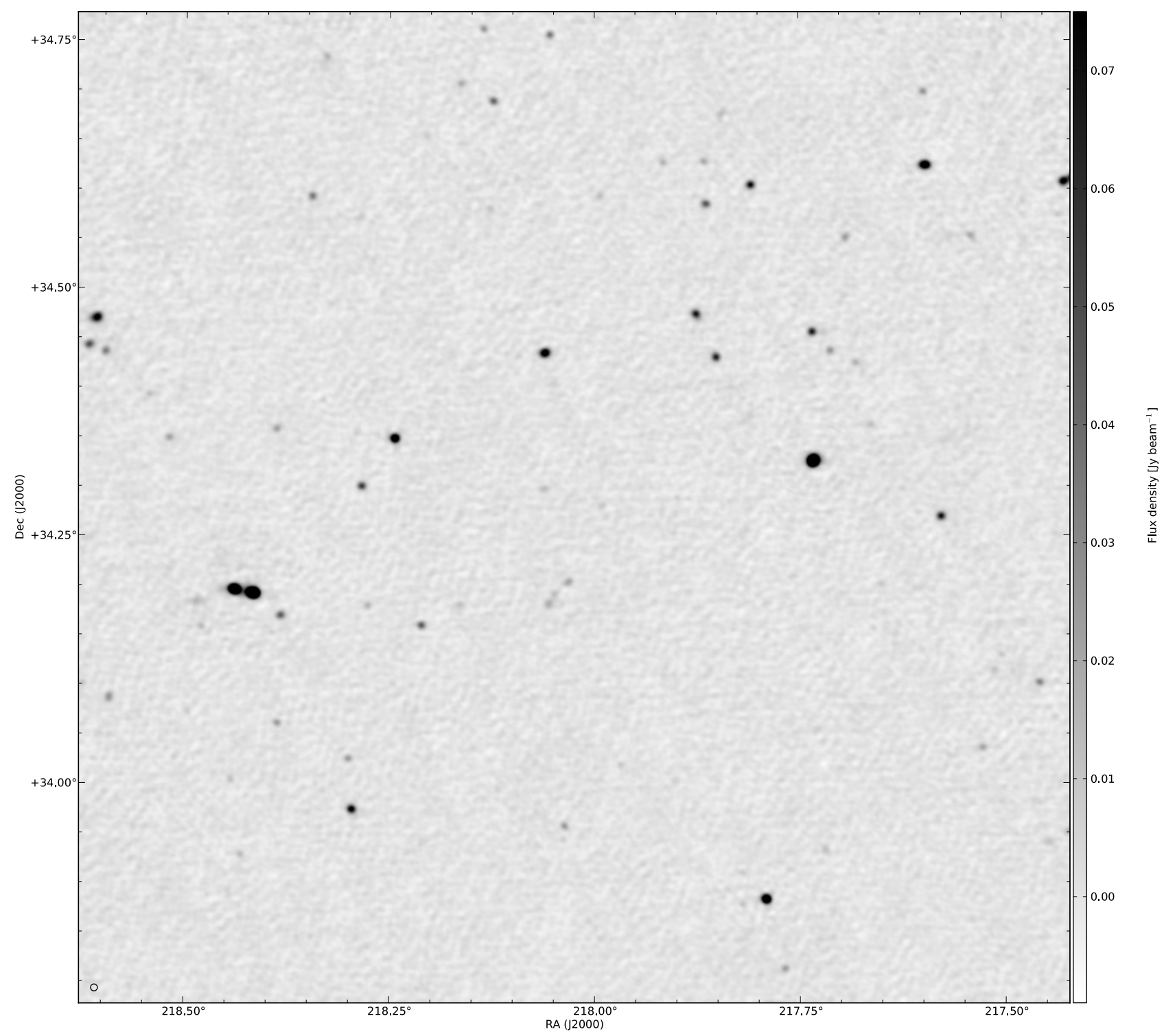

Fig. 3. Zoom-in of the central part of the mosaic. The image covers 1 square degree. The greyscale shows the flux density from $-3 \sigma_{\text {avg }}$ to $25 \sigma_{\text {avg }}$ where $\sigma_{\text {avg }}=3.0 \mathrm{mJy}^{-1}$ beam ${ }^{-1}$ is the average rms across the entire mosaic.

The final catalogue consists of 1289 sources between $4.1 \mathrm{mJy}$ and $7.3 \mathrm{Jy}$ and is available as part of the online version of this article (Table A.1) and from the $\mathrm{CDS}^{3}$. The flux scales of the individual pointings were adjusted prior to mosaicing as described in Sect. 3.4 and the astrometry in the catalogue has been corrected for a systematic offset also described in Sect. 3.4. A sample of the catalogue is shown in Table 4 where the columns are: (1) Source name; $(2,3)$ flux-weighted position right ascension, RA, and uncertainty; $(4,5)$ flux-weighted position declination, Dec, and uncertainty; (6) integrated source flux density and uncertainty; (7) peak flux density and uncertainty; (8-10) fitted parameters: deconvolved major- and minor-axes, and position angle, for extended sources; (11) local rms noise; and (12) the number of Gaussians fitted to the source. Extended sources are classified as such based on the ratio between the integrated and peak flux densities (see Sect. 3.2). Unresolved sources have a "-" listed for all their fitted shape parameters (semi-major and -minor axes and position angle) or for only the semi-minor axis where the source

3 http://cdsweb.u-strasborg.fr/ is resolved in one direction. For extended sources consisting of multiple Gaussians, the fitted parameters for each Gaussian are given on separate lines in the table, listed as "a", "b", etc. Images of the 25 brightest sources are shown in Appendix A.

\subsection{Resolved sources}

In the presence of no noise, the extendedness of a source can simply be determined from the ratio of the integrated flux density to the peak flux density, $S_{\mathrm{i}} / S_{\mathrm{p}}>1$. However, since the errors on $S_{\mathrm{i}}$ and $S_{\mathrm{p}}$ are correlated, the $S_{\mathrm{i}} / S_{\mathrm{p}}$ distribution is skewed, particularly at low signal-to-noise. To determine an upper envelope of this distribution, we performed a Monte-Carlo simulation in which we generated 25 random fields containing $\sim 10000$ randomly positioned point sources with peak flux densities between $0.1 \sigma$ and $20 \sigma$, where $\sigma$ was taken to be $3 \mathrm{mJy}^{\text {beam }}{ }^{-1}$. The source flux densities are drawn randomly from the source count distribution, $\mathrm{d} N / \mathrm{d} S \propto S^{-1.6}$ (Sect. 4.1). We neglect the deviation of the true source counts from a power law slope at high fluxes as there are very few sources at these fluxes. 
A\&A 549, A55 (2013)

Table 4. Sample of the GMRT $153 \mathrm{MHz}$ source and Gaussian-component catalogue.

\begin{tabular}{|c|c|c|c|c|c|c|c|c|c|c|c|}
\hline $\begin{array}{c}\text { Source ID } \\
\text { (1) }\end{array}$ & $\begin{array}{l}\text { RA } \\
\text { [deg] } \\
(2)\end{array}$ & $\begin{array}{c}\sigma_{\mathrm{RA}} \\
{\left[{ }^{\prime \prime}\right]} \\
(3)\end{array}$ & $\begin{array}{c}\text { Dec } \\
{[\mathrm{deg}]} \\
(4)\end{array}$ & $\begin{array}{c}\sigma_{\text {Dec }} \\
{\left[{ }^{\prime \prime}\right]} \\
(5)\end{array}$ & $\begin{array}{c}S_{\mathrm{i}} \\
{[\mathrm{mJy}]} \\
(6)\end{array}$ & $\begin{array}{c}S_{\mathrm{p}} \\
{\left[\mathrm{mJy} \mathrm{beam}^{-1}\right]} \\
(7)\end{array}$ & $\begin{array}{l}a^{a} \\
{\left[{ }^{\prime \prime}\right]} \\
(8)\end{array}$ & $\begin{array}{l}b^{a} \\
{["]} \\
(9)\end{array}$ & $\begin{array}{c}\phi^{a} \\
{[\mathrm{deg}]} \\
(10)\end{array}$ & $\begin{array}{c}\mathrm{rms} \\
{\left[\mathrm{mJy} \mathrm{beam}^{-1}\right]} \\
(11)\end{array}$ & $\begin{array}{r}N_{\text {Gauss }}{ }^{b} \\
(12)\end{array}$ \\
\hline $\mathrm{J} 144733+3507$ & 221.88796 & 0.9 & 35.13126 & 1.5 & $158 \pm 33$ & $69 \pm 15$ & $38.3 \pm 3.4$ & $19.3 \pm 1.9$ & $5 \pm 6$ & 5.4 & 1 \\
\hline $\mathrm{J} 144658+3308$ & 221.74358 & 2.9 & 33.14058 & 1.1 & $42 \pm 13$ & $20 \pm 6$ & & & & 4.9 & 2 \\
\hline $\mathrm{a}$ & 221.74843 & 2.8 & 33.14096 & 1.8 & $19 \pm 8$ & $19 \pm 5$ & - & - & - & & \\
\hline $\mathrm{b}$ & 221.73914 & 2.8 & 33.14001 & 2.6 & $23 \pm 8$ & $18 \pm 5$ & $15.9 \pm 6.8$ & $9.5 \pm 5.6$ & $56 \pm 90$ & & \\
\hline $\mathrm{J} 144705+3442$ & 221.77266 & 1.8 & 34.71605 & 1.6 & $32 \pm 9$ & $27 \pm 7$ & - & - & - & 4.7 & 1 \\
\hline $\mathrm{J} 144706+3457$ & 221.77552 & 1.4 & 34.95096 & 1.5 & $25 \pm 9$ & $28 \pm 7$ & - & - & - & 5.0 & 1 \\
\hline $\mathrm{J} 144645+3330$ & 221.69153 & 1.8 & 33.50822 & 1.4 & $31 \pm 9$ & $29 \pm 7$ & - & - & - & 4.8 & 1 \\
\hline $\mathrm{J} 144640+3322$ & 221.66919 & 0.9 & 33.36920 & 0.8 & $72 \pm 16$ & $62 \pm 13$ & - & - & - & 4.9 & 1 \\
\hline $\mathrm{J} 144646+3440$ & 221.69543 & 1.5 & 34.67482 & 1.1 & $29 \pm 9$ & $32 \pm 8$ & - & - & - & 5.1 & 1 \\
\hline $\mathrm{J} 144648+3546$ & 221.70230 & 1.9 & 35.77421 & 1.4 & $19 \pm 8$ & $25 \pm 6$ & - & - & - & 5.0 & 1 \\
\hline $\mathrm{J} 144613+3303$ & 221.55831 & 0.7 & 33.06494 & 0.6 & $169 \pm 35$ & $131 \pm 27$ & - & - & - & 5.0 & - \\
\hline $\mathrm{J} 144638+3553$ & 221.65996 & 2.1 & 35.88845 & 2.3 & $37 \pm 11$ & $27 \pm 7$ & - & - & - & 5.7 & 1 \\
\hline $\mathrm{J} 144626+3512$ & 221.61124 & 1.0 & 35.20927 & 0.7 & $314 \pm 65$ & $244 \pm 50$ & - & - & - & 7.5 & 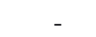 \\
\hline $\mathrm{J} 144619+3425$ & 221.58123 & 3.2 & 34.42447 & 2.4 & $46 \pm 12$ & $26 \pm 7$ & - & - & - & 5.5 & 1 \\
\hline $\mathrm{J} 144606+3316$ & 221.52865 & 2.9 & 33.26922 & 2.2 & $20 \pm 7$ & $17 \pm 5$ & - & - & - & 4.2 & 1 \\
\hline $\mathrm{J} 144557+3251$ & 221.49062 & 0.6 & 32.86032 & 0.5 & $122 \pm 26$ & $111 \pm 23$ & - & - & - & 5.0 & 1 \\
\hline $\mathrm{J} 144555+3237$ & 221.48098 & 2.4 & 32.62328 & 3.6 & $24 \pm 7$ & $17 \pm 5$ & - & - & - & 4.4 & 1 \\
\hline $\mathrm{J} 144617+3506$ & 221.57405 & 1.1 & 35.11639 & 1.3 & $158 \pm 34$ & $75 \pm 16$ & & & & 5.3 & 2 \\
\hline $\mathrm{a}$ & 221.57091 & 0.9 & 35.11314 & 0.7 & $93 \pm 20$ & $78 \pm 16$ & $15.3 \pm 1.8$ & $5.6 \pm 1.4$ & $72 \pm 16$ & & \\
\hline b & 221.57923 & 1.4 & 35.12223 & 2.1 & $65 \pm 15$ & $38 \pm 9$ & $29.4 \pm 5.1$ & $10.6 \pm 2.8$ & $12 \pm 14$ & & \\
\hline $\mathrm{J} 144602+3339$ & 221.50949 & 2.1 & 33.66347 & 1.3 & $42 \pm 10$ & $27 \pm 6$ & $29.3 \pm 5.3$ & - & $64 \pm 11$ & 3.8 & 1 \\
\hline $\mathrm{J} 144607+3503$ & 221.52967 & 1.2 & 35.05975 & 0.6 & $81 \pm 18$ & $62 \pm 13$ & - & - & $178 \pm 6$ & 4.8 & 1 \\
\hline
\end{tabular}

Notes. ${ }^{(a)}$ Parameters are given for extended sources to which Gaussian components were successfully fit. ${ }^{(b)}$ A “-” indicates a poor Gaussian fit. In these cases the total flux density quoted is the total flux density in the source island.
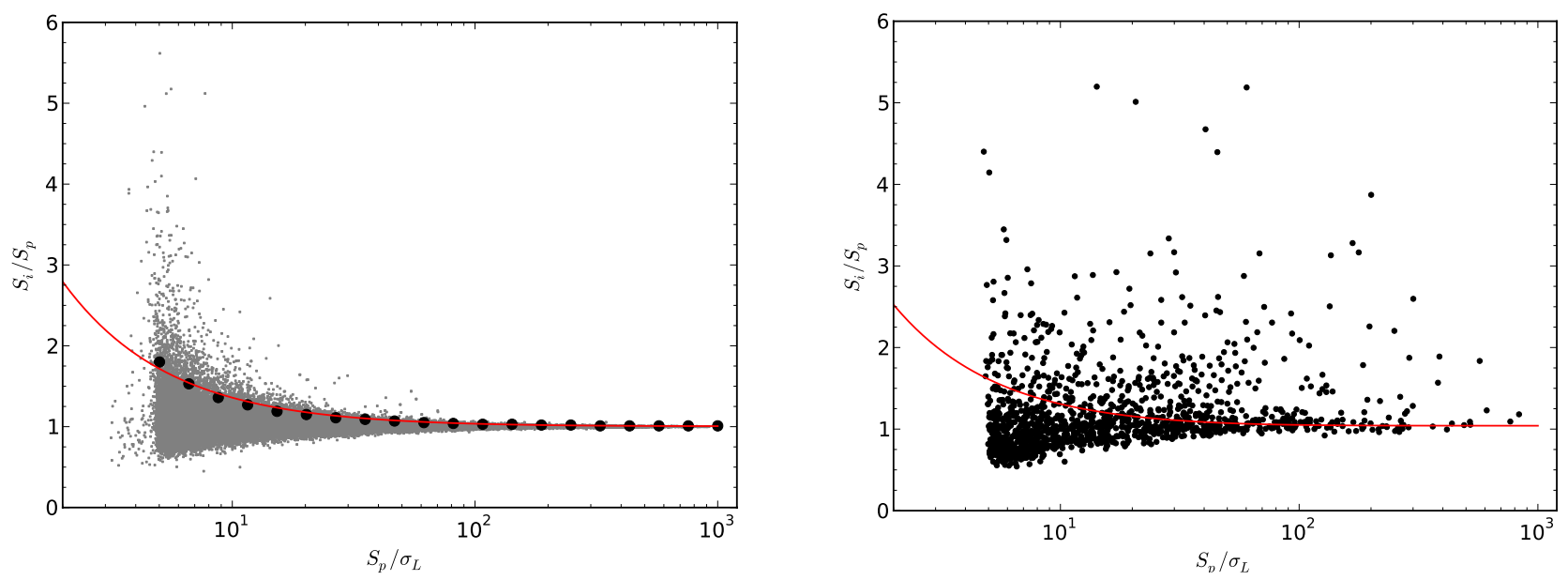

Fig. 4. Left: the simulated ratio of integrated to peak flux density as a function of signal-to-noise ratio for sources from the 25 Monte-Carlo simulations. For 20 logarithmic bins in signal-to-noise ratio, the black points show the threshold below which 95 per cent of the sources lie in that bin. The red line shows a fit to this upper envelope. Right: the measured ratio of integrated to peak flux density as a function of signal-to-noise ratio. The line shows the upper envelope containing 95 per cent of the unresolved sources as determined from Monte-Carlo simulations.

The rms noise map for these fields was taken from the central $4000 \times 4000$ pixel $^{2}$ of the residual mosaic. Source detection was performed in the same manner described in Sect. 3.1, thus only $\sim 750$ sources in each field satisfy the detection criterion of peak flux density $>5 \sigma$. The $S_{\mathrm{i}} / S_{\mathrm{p}}$ distribution produced from the Monte-Carlo simulation is plotted in the left panel of Fig. 4. To determine the 95 per cent envelope, a curve was fit to the 95th percentile of 20 logarithmic bins across signal-to-noise ratio. The fitted envelope is characterised by:

$S_{\mathrm{i}} / S_{\mathrm{p}}=1+\left\{(0.01 \pm 0.02)^{2}+(3.58 \pm 0.10)^{2}\left(\sigma_{\mathrm{L}} / S_{\mathrm{p}}\right)^{2}\right\}^{0.5}$.

The measured distribution of $S_{\mathrm{i}} / S_{\mathrm{p}}$ as a function of signalto-noise ratio is shown in the right panel of Fig. 4. The line shows the upper envelope from the Monte-Carlo simulation.
Of the 453 sources that lie above this line (35 per cent of all 1289 sources), approximately 41, i.e. 9 per cent, are not truly extended and merely lie above the line by chance. However, all these sources above the line are listed in the catalogue as extended and the measured deconvolved FWHM major and minor axes are given.

\subsection{Completeness and reliability}

To quantify the completeness and reliability of the catalogue, we performed a similar Monte-Carlo simulation to that described in the previous section. However, in this case approximately 25 per cent of the artificial sources inserted into the noise map were extended sources - Gaussians with FWHM larger than 
W. L. Williams et al.: T-RaMiSu: The Two-meter Radio Mini Survey. I.
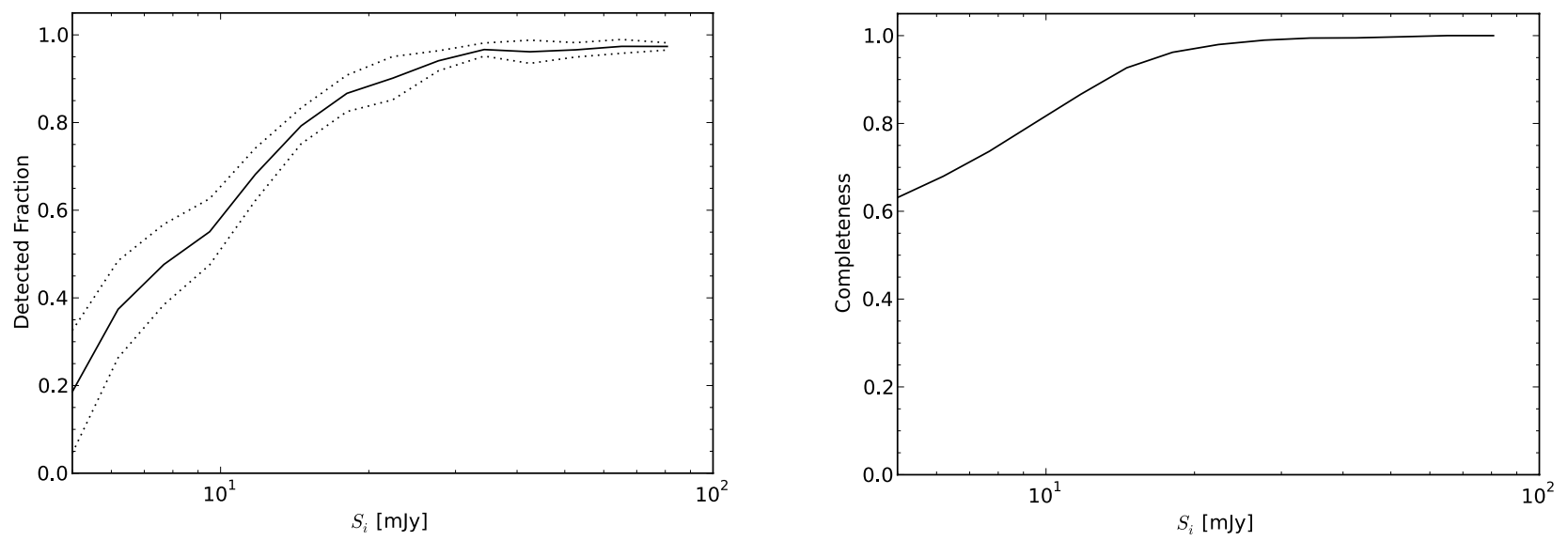

Fig. 5. Left: fraction of sources detected as a function of integrated flux density to local noise ratio calculated from 25 Monte-Carlo simulations. The solid line shows the mean of all 25 randomly generated fields and the two dotted lines show the $1 \sigma$ uncertainty. Right: estimated completeness of the catalogue as a function of integrated flux density limit accounting for the varying sensitivity across the field of view.
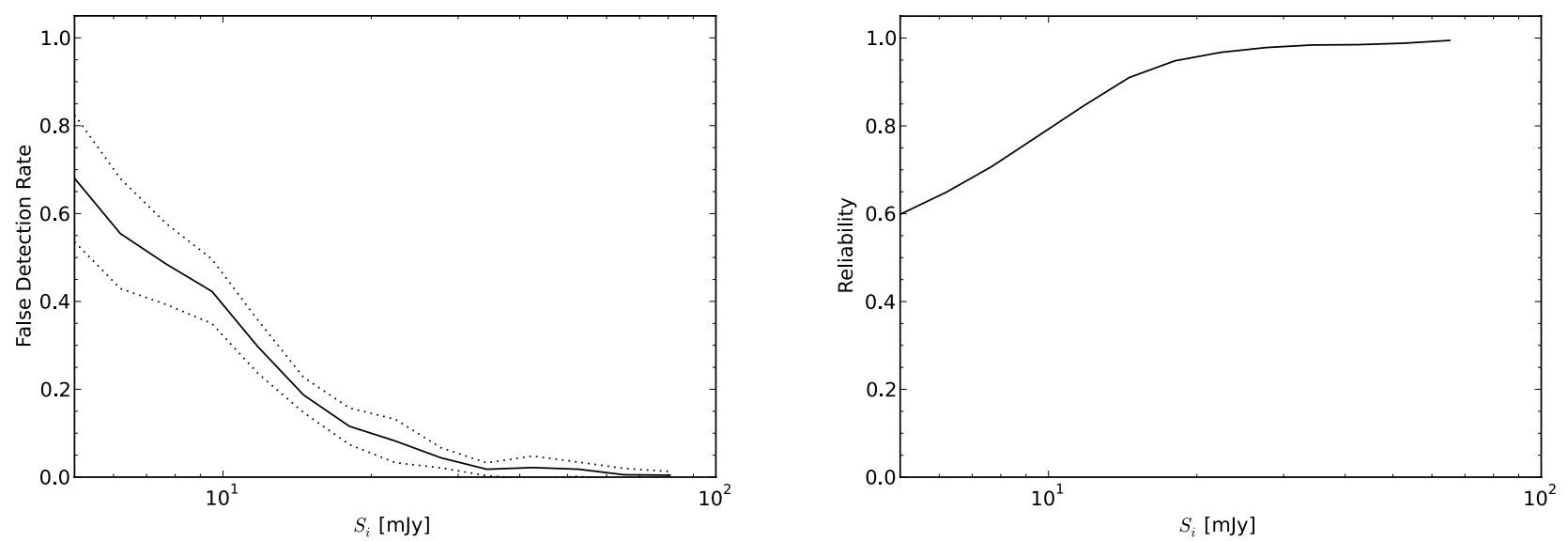

Fig. 6. Left: false detection rate as a function of peak flux density to local signal-to-noise ratio calculated from 25 Monte-Carlo simulations. The solid line shows the mean of all 25 randomly generated fields and the two dotted lines show the $1 \sigma$ uncertainty. Right: estimated reliability of the catalogue as a function of integrated flux density limit accounting for the varying sensitivity across the field of view.

the beamsize. This allows for a better estimate of the completeness and reliability in terms of integrated flux densities.

The completeness of a catalogue represents the probability that all sources above a given flux density are detected. We have estimated this by plotting the fraction of detected sources in our MC simulation as a function of integrated flux density (left panel of Fig. 5), i.e. the fraction of input sources that have a catalogued flux density using the same detection parameters. Due to the variation in rms across the image, the detection fraction has been multiplied by the fraction of the total $30 \mathrm{deg}^{2}$ area in which the source can be detected. The completeness at a given flux density is determined by integrating the detected fraction upwards from a given flux density limit and is plotted as a function of integrated flux density in the right panel of Fig. 5. We thus estimate that the catalogue is 95 per cent complete above a peak flux density of $14 \mathrm{mJy}$.

The reliability of the catalogue indicates the probability that all sources above a given flux density are real. In the left panel of Fig. 6, the false detection rate $F D R$, i.e. the fraction of catalogued sources that do not have an input source, is plotted as a function of the integrated flux density. Integrating up from a given detection limit and multiplying by the normalised source flux distribution, we can determine an estimate of the overall FDR or reliability, $R=1-F D R$, of the catalogue. The reliability is plotted as a function of integrated flux density limit in the right panel of Fig. 6. For a 14 mJy detection threshold, the reliability is 92 per cent.

\subsection{Astrometric and flux uncertainties}

Errors in the phase calibration introduce uncertainties in the source positions. To assess these uncertainties and determine any systematic offsets we selected a sample of sources with peak flux densities at least $10 \sigma_{\mathrm{L}}$. We searched for $1.4 \mathrm{GHz}$ NVSS (Condon et al. 1998) sources within $45^{\prime \prime}$ of these targets. 745 matches were found. From this sample, we measured a small offset of $(\Delta \alpha, \Delta \delta)=\left(0.44^{\prime \prime},-0.21^{\prime \prime}\right)$, which is of the order of the pixel size of the $153 \mathrm{MHz}$ observations and the NVSS accuracy $\left(\sim 1^{\prime \prime}\right)$. A correction for this offset has been applied to all sources in the catalogue. The scatter in the offsets between the GMRT and NVSS positions is a combination of noise-independent calibration errors, $\epsilon$, in both the GMRT and NVSS data as well as a noise-dependent error, $\sigma$, from position determination via Gaussian-fitting:

$\sigma^{2}=\epsilon_{\mathrm{GMRT}}^{2}+\epsilon_{\mathrm{NVSS}}^{2}+\sigma_{\mathrm{GMRT}}^{2}+\sigma_{\mathrm{NVSS}}^{2}$.

From Condon et al. (1998), the NVSS calibration errors are $\left(\epsilon_{\alpha}, \epsilon_{\delta}\right)_{\mathrm{NVSS}}=\left(0.45^{\prime \prime}, 0.56^{\prime \prime}\right)$. To separate the noise-dependent and -independent uncertainties we select from the above sample 
only the NVSS sources with position errors of less than $0.6^{\prime \prime}$ and measure a scatter of $\left(\sigma_{\alpha}, \sigma_{\delta}\right)_{\mathrm{GMRT}}=\left(0.67^{\prime \prime}, 0.65^{\prime \prime}\right)$. For this very high signal-to-noise sub-sample of 107 sources the noise-dependent fit errors for both the GMRT and NVSS can safely be assumed to be small so we determine the GMRT calibration errors to be $\left(\epsilon_{\alpha}, \epsilon_{\delta}\right)_{\mathrm{GMRT}}=\left(0.50^{\prime \prime}, 0.32^{\prime \prime}\right)$. These are added quadratically to the Gaussian-fit position uncertainties in the catalogue.

Similarly, in addition to the noise-dependent Gaussian fitting uncertainties on the fluxes (Condon 1997), the uncertainty in the measured flux densities also consists of a noise-independent component. The uncertainty introduced through transferring the flux density scale from the calibrator to the target fields is the main such uncertainty and depends on a number of factors: (i) the data quality; (ii) the accuracy of the model; and (iii) differences in observing conditions between the calibrator and target.

Like the target data, the calibrator data is adversely affected by RFI and the ionosphere. The RFI conditions of the flanking field observations were similar to those when the central pointing data were taken, however, the ionosphere was not as calm. Following Intema et al. (2011) we adopt a slightly inflated, ad-hoc amplitude uncertainty of $\sim 4$ per cent due to RFI and ionospheric effects.

The calibrator model is of a point source whose flux density at $153 \mathrm{MHz}$ is predicted from the Perley-Taylor model based on flux density measurements at many frequencies. 3C 48 is a point source of $64.4 \mathrm{Jy}$ at $153 \mathrm{MHz}$. Intema et al. (2011) provide an improved model for $3 \mathrm{C} 286$, a point source of $31.01 \mathrm{Jy}$ at $153 \mathrm{MHz}$, and estimate a flux density uncertainty of 5 per cent. The large field of view, however, means that there are other fainter sources present in the calibrator field. For similar duration observations of 3C 286 Intema et al. (2011) set an upper limit of 1 per cent on the flux density uncertainty. Since $3 \mathrm{C} 48$ is about a factor of two brighter, we estimate that the flux density uncertainty due to additional sources in the $3 \mathrm{C} 48$ field is also at most 1 per cent.

Individual antennas are sensitive to the galactic diffuse radio emission which varies across the sky and so may be different for the calibrator and target fields thereby introducing an offset to the flux density scale as well as additional uncertainty. However, since the GMRT does not measure the sky temperature, we require external information to take this into account. Following Tasse et al. (2007) and Intema et al. (2011) we determine the mean off-source sky-temperature from the Haslam et al. (1982) all-sky radio maps at $408 \mathrm{MHz}$ : both the Boötes and 3C 286 fields have sky temperatures of $\sim 20 \pm 1 \mathrm{~K}$. Using the equation from Tasse et al. (2007), this implies that no offset in the flux density scale is required for 3C 286 and we estimate a gain uncertainty of 2 per cent. However, the sky temperature near the primary calibrator $3 \mathrm{C} 48$ is $24 \pm 1 \mathrm{~K}$ which implies a flux density correction of 0.92 with an estimated uncertainty of 8 per cent. Since the flux density scale is linear, this offset is applied post hoc to the measured flux densities.

Prior to combining the individual pointings, we compared the measured primary beam-corrected flux densities of sources in the overlapping regions (approximately 110-150 sources per region) and found those in the flanking fields to be consistently higher by $30 \pm 5$ per cent. To investigate this we made images after calibration using 3C 286 as the primary calibrator. This yielded consistent fluxes between the central and flanking fields. It is likely that significant time-dependent changes in the GMRT systems over the course of each observing night were captured by the regular (each $30 \mathrm{~min}$ ) observations of 3C 286 . We thus used the 3C 286-calibrated images to derive a correction
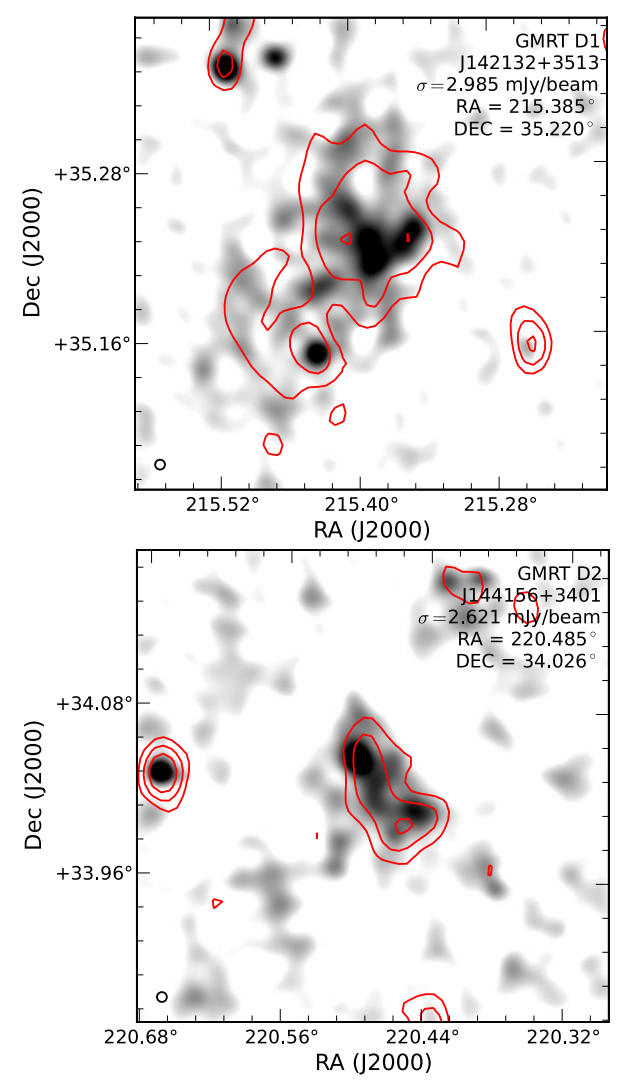

Fig. 7. Postage stamps showing D1, RA $=14: 21: 32$, Dec $=+35: 12: 12$ (top) and D2, RA $=14: 41: 56$, Dec $=+34: 01: 34$ (bottom). The greyscale goes from $0.5 \sigma$ to $5 \sigma$ and the images have been smoothed with a Gaussian of 50 arcsec. WENSS contours are overlaid at $[1.5,3.0,10.0] \times \sigma_{\mathrm{L}}$ where $\sigma_{\mathrm{L}}$ is the local $\mathrm{rms}$ in the WENSS images -3.5 mJy beam $^{-1}$ and $3.7 \mathrm{mJy}_{\text {beam }}^{-1}$ respectively for D1 and D2.

to the flux density scales of the flanking fields, a factor of 1.3, before combining the individual pointings. The uncertainty of this correction is 10 per cent.

The total estimated uncertainty in transferring the flux density scale is of the order of 20 per cent which we add quadratically to the measured Gaussian fit uncertainty for each source. Comparison of the flux density of bright sources measured in the individual pointings after the above correction shows good agreement between the flux density scales of the individual pointings and the measured scatter is $\sim 16$ per cent, which also includes a contribution by the noise-dependent terms.

\subsection{Diffuse sources}

We have identified two faint diffuse sources in the final mosaic which were not detected by PyBDSM as their peak flux densities are too low. Postage stamps of these two sources are shown in Fig. 7. The first, D1, is located at RA $=14: 21: 32$, Dec $=+35: 12: 12$. This source has previously been detected in WENSS by Delain \& Rudnick (2006) who have associated it with a galaxy group at $z=0.01$. The second diffuse source, D2, is located at $\mathrm{RA}=14: 41: 56, \mathrm{Dec}=+34: 01: 34$.

\section{Analysis}

The 1289 sources in the catalogue provide a statistically significant sample across three orders of magnitude in flux density from $4 \mathrm{mJy}$ to $7 \mathrm{Jy}$. In this section we present the derived $153 \mathrm{MHz}$ source counts and spectral index distributions 
W. L. Williams et al.: T-RaMiSu: The Two-meter Radio Mini Survey. I.

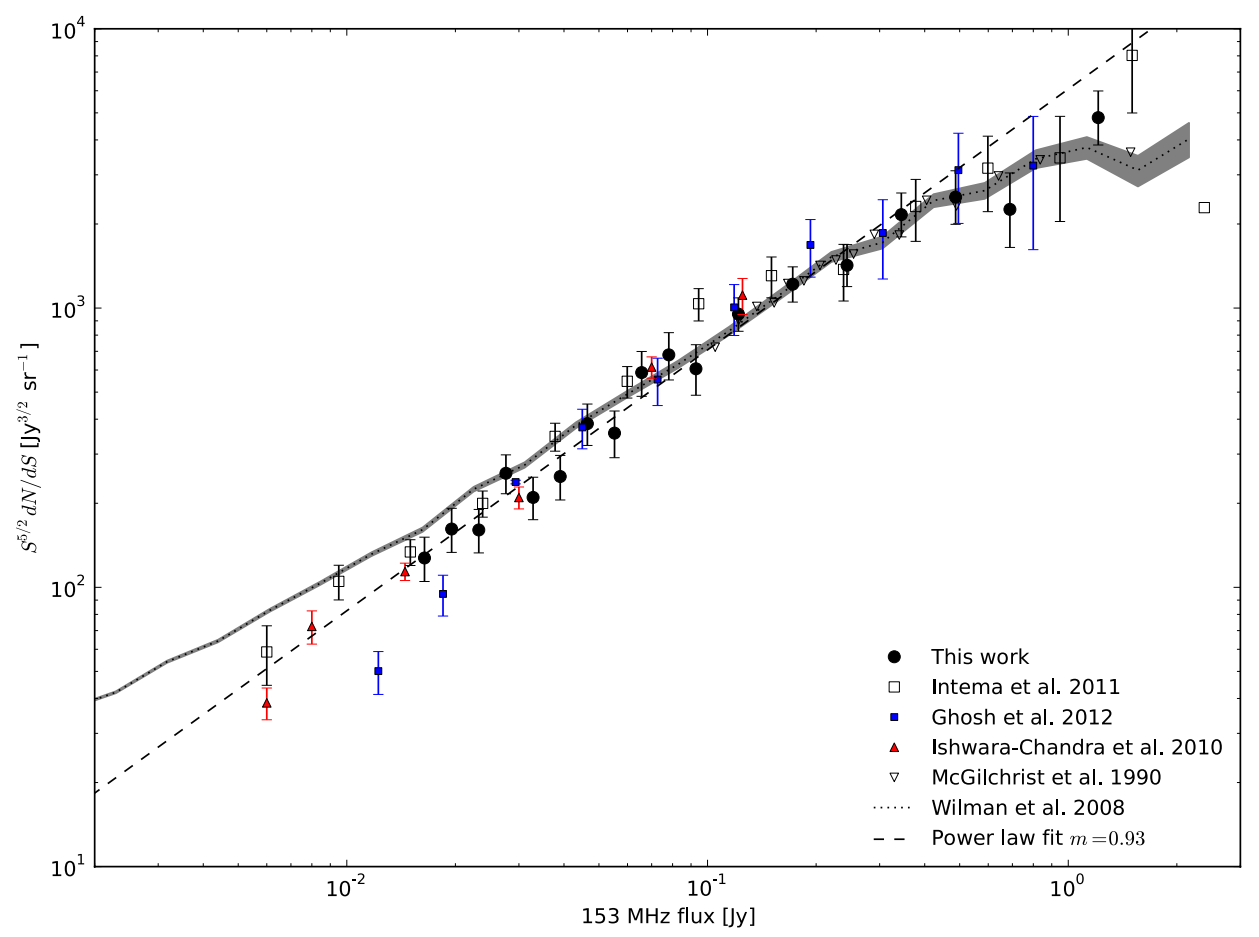

Fig. 8. Euclidean-normalised differential source counts for the GMRT $153 \mathrm{MHz}$ catalogue (filled black circles) in 18 logarithmic flux density bins between $15 \mathrm{mJy}$ and $6.5 \mathrm{Jy}$. For comparison we have plotted the $153 \mathrm{MHz}$ source counts from Intema et al. (2011) (open squares) for the central Böotes pointing, from Ghosh et al. (2012) (blue filled squares) and from Ishwara-Chandra et al. (2010) (red filled triangles), as well as the $151 \mathrm{MHz}$ source counts from McGilchrist et al. (1990) for part of the 7C catalogue (open inverted triangles). Also shown is a source count model by Wilman et al. (2008) (dotted line with shaded area indicating the $1 \sigma$ errors) and a power law fitted between $150-400 \mathrm{mJy}$ (dashed line) which has a slope of $0.93 \pm 0.04$.

based on matching these sources to catalogues at $1.4 \mathrm{GHz}$ and $320 \mathrm{MHz}$.

\subsection{Source counts}

The Euclidean-normalised differential source counts are shown in Fig. 8. Due to the large variation in rms across the mosaic, the sources are not uniformly detected across the image, i.e. faint sources can only be detected in a smaller area in the inner part of the image. We therefore weight each source by the inverse of the area in which it can be detected (e.g. Windhorst et al. 1985), which also accounts for the varying detection area within a given flux density bin. Accurate derivation of the source counts is complicated by a number of effects. In general, noise can scatter sources into adjacent bins, most noticably at low flux densities. A positive bias is introduced by the enhancement of weak sources by random noise peaks (Eddington bias). Furthermore, low surface brightness extended sources can be missed as their peak flux densities fall below the detection limit. We have used our Monte-Carlo simulations to estimate the combined contribution of these effects and derive a correction factor to the observed source counts. Errors on the final normalised source counts are propagated from the errors on the correction factors and the Poisson errors (Gehrels 1986) on the raw counts per bin. The flux density bins start at three times the average rms, $15 \mathrm{mJy}$, and increase in factors of $2^{1 / 4}, 2^{1 / 2}$ or 2 chosen to provide source counts of 60-80 in most, except for the highest, flux density bins. Table 5 lists (i) the flux density bins; (ii) the central flux density of the bin; (iii) the raw counts; (iv) the effective detection areas for sources at the lower and upper limits of the flux density bin; (v) the effective area corresponding to the bin centre; (vi) the mean weight of the sources in the bin; (vii) the correction factor; and (viii) the corrected normalised source counts.
We have compared our source counts with the little observational data available at this frequency. Our source counts agree well with those derived by Intema et al. (2011) for the central field. Since their image is approximately three times deeper than our mosaic, the good agreement at low flux densities lends credance to our correction factors. The recent source counts from Ghosh et al. (2012) and those by Ishwara-Chandra et al. (2010) for a smaller, slightly shallower GMRT field also agree well with our data, except the Ghosh et al. (2012) counts deviate at low flux densities, becoming increasingly lower. At the high flux end, the 7C $151 \mathrm{MHz}$ source counts (McGilchrist et al. 1990) match our counts well. We have fit a power law over the flux density range $15-400 \mathrm{mJy}$ and obtain a slope of $0.93 \pm 0.04$ which is consistent with, but slightly steeper than, the 0.91 obtained by Intema et al. (2011) across the same flux density range. Likewise, it is consistent with the value of 1.01 found by Ishwara-Chandra et al. (2010), but is slightly shallower. The source counts derived from the small sample of George \& Stevens (2008) (not plotted) are fit by a single power law with a slope of 0.72 , but their deviation is probably due to poor statistics. Model source counts have been derived by Wilman et al. (2008) for the $151 \mathrm{MHz}$ source population predicted from the extrapolated radio luminosity functions of different radio sources in a $\Lambda \mathrm{CDM}$ framework. The Wilman et al. (2008) model catalogue has been corrected with their recommended post-processing, which effectively reduces the source count slightly at low flux densities. The dominant source population at flux densities above $\sim 200 \mathrm{mJy}$ is that of FRII radio sources. Only below this flux density does the FRI population begin to dominate. There is a general agreement between our data and this model which has an approximate power-law slope of 0.79 between 10 and $400 \mathrm{mJy}$. At low flux densities it is likely that the Wilman et al. (2008) counts slightly overestimate the 
Table 5. Euclidean-normalised differential source counts for the GMRT $153 \mathrm{MHz}$ catalogue.

\begin{tabular}{cccccccc}
\hline \hline $\begin{array}{c}S \text { Range } \\
{[\mathrm{Jy}]}\end{array}$ & $\begin{array}{c}S_{\mathrm{c}} \\
{[\mathrm{Jy}]}\end{array}$ & Raw counts & $\begin{array}{c}\text { Area } \\
{\left[\mathrm{deg}^{2}\right]}\end{array}$ & $\begin{array}{c}A\left(S_{\mathrm{c}}\right) \\
{\left[\mathrm{deg}^{2}\right]}\end{array}$ & $\langle W\rangle$ & Correction & $\begin{array}{c}\text { Normalised counts } \\
{\left[\mathrm{Jy}^{3 / 2} \mathrm{sr}^{-1}\right]}\end{array}$ \\
\hline $0.015-0.018$ & 0.016 & $70.0_{-8.3}^{+9.4}$ & $17.1-22.1$ & 19.7 & 0.80 & $1.14 \pm 0.15$ & $127_{-22}^{+24}$ \\
$0.018-0.021$ & 0.020 & $68.0_{-8.2}^{+9.3}$ & $22.1-26.5$ & 24.4 & 0.83 & $1.11 \pm 0.14$ & $162_{-28}^{+30}$ \\
$0.021-0.025$ & 0.023 & $66.0_{-8.1}^{+9.2}$ & $26.5-30.5$ & 28.7 & 0.91 & $1.06 \pm 0.13$ & $160_{-28}^{+30}$ \\
$0.025-0.030$ & 0.028 & $86.0_{-9.3}^{+10.3}$ & $30.5-32.9$ & 32.1 & 0.93 & $1.00 \pm 0.11$ & $256_{-40}^{+42}$ \\
$0.030-0.036$ & 0.033 & $62.0_{-7.9}^{+8.9}$ & $32.9-33.3$ & 33.2 & 0.96 & $0.94 \pm 0.10$ & $210_{-35}^{+38}$ \\
$0.036-0.042$ & 0.039 & $58.0_{-7.6}^{+8.7}$ & $\ldots$ & 33.3 & 0.95 & $0.90 \pm 0.11$ & $249_{-44}^{+47}$ \\
$0.042-0.050$ & 0.046 & $74.0_{-8.6}^{+9.6}$ & $\ldots$ & 33.3 & 0.99 & $0.91 \pm 0.11$ & $386_{-64}^{+68}$ \\
$0.050-0.060$ & 0.055 & $51.0_{-7.1}^{+8.2}$ & $\ldots$ & 33.3 & 0.99 & $0.93 \pm 0.11$ & $357_{-65}^{+71}$ \\
$0.060-0.071$ & 0.066 & $64.0_{-8.0}^{+9.0}$ & $\ldots$ & 33.3 & 0.99 & $0.95 \pm 0.12$ & $588_{-105}^{+112}$ \\
$0.071-0.085$ & 0.078 & $57.0_{-7.5}^{+8.6}$ & $\ldots$ & 33.3 & 1.00 & $0.96 \pm 0.13$ & $680_{-127}^{+137}$ \\
$0.085-0.101$ & 0.093 & $39.0_{-6.2}^{+7.3}$ & $\ldots$ & 33.3 & 1.00 & $0.96 \pm 0.11$ & $606_{-119}^{+133}$ \\
$0.101-0.143$ & 0.122 & $81.0_{-9.0}^{+10.0}$ & $\ldots$ & 33.3 & 1.00 & $0.96 \pm 0.07$ & $951_{-126}^{+136}$ \\
$0.143-0.202$ & 0.172 & $61.0_{-7.8}^{+8.9}$ & $\ldots$ & 33.3 & 1.00 & $0.97 \pm 0.05$ & $1218_{-167}^{+187}$ \\
$0.202-0.285$ & 0.244 & $42.0_{-6.5}^{+7.5}$ & $\ldots$ & 33.3 & 1.00 & $0.98 \pm 0.05$ & $1423_{-229}^{+265}$ \\
$0.285-0.404$ & 0.345 & $38.0_{-6.1}^{+7.2}$ & $\ldots$ & 33.3 & 1.00 & $0.98 \pm 0.04$ & $2160_{-362}^{+422}$ \\
$0.404-0.571$ & 0.487 & $26.0_{-5.1}^{+6.2}$ & $\ldots$ & 33.3 & 1.00 & $0.98 \pm 0.05$ & $2496_{-502}^{+606}$ \\
$0.571-0.807$ & 0.689 & $14.0_{-3.7}^{+4.8}$ & $\ldots$ & 33.3 & 1.00 & $0.98 \pm 0.05$ & $2257_{-608}^{+790}$ \\
$0.807-1.615$ & 1.211 & $25.0_{-5.0}^{+6.1}$ & $\ldots$ & 33.3 & 1.00 & $0.98 \pm 0.04$ & $4807_{-971}^{+1182}$ \\
$1.615-6.458$ & 4.036 & $9.0_{-2.9}^{+4.1}$ & $\ldots$ & 33.3 & 1.00 & $0.99 \pm 0.01$ & $5925_{-1936}^{+2715}$ \\
\hline
\end{tabular}

true counts due to double counting of hybrid AGN-star forming galaxies.

\subsection{Spectral index distributions}

While deep 1.4 GHz data exists for the Boötes Field (de Vries et al. 2002), this only covers the central $7 \mathrm{deg}^{2}$. This data was used in Intema et al. (2011) in a $153 \mathrm{MHz}$ flux-limited spectral index analysis. However, we choose to compare our source list to the NVSS 1.4 GHz catalogue (Condon et al. 1994) which covers our entire survey area at a comparable resolution. We searched for NVSS counterparts within $45^{\prime \prime}$ of each GMRT source. Despite the relatively small difference in resolution between the NVSS (45") and the GMRT (25") data, a small number of GMRT sources ( 9 pairs) were matched the same NVSS source. Also, due to differences in the grouping of components into sources, we merged 16 pairs of NVSS sources which matched a single GMRT source. Sources were merged by summing their total flux densities. A spectral index was calculated for each GMRT source based on the combined flux density of merged sources.

We matched 1134 NVSS sources to 1127 GMRT sources and then used this matched subsample to compute the spectral in$\operatorname{dex}^{4}$ distribution which is shown in Fig. 9. The flux density limit of $2.5 \mathrm{mJy}$ at $1.4 \mathrm{GHz}$ biases the detection of $1.4 \mathrm{GHz}$ counterparts to fainter $153 \mathrm{MHz}$ sources to those with flatter spectra. 168 GMRT sources have no match in NVSS. These are consistent with having steeper spectral indices below the diagonal line in Fig. 9 and we therefore provide an an upper limit to the spectral index given the NVSS flux density limit. The mean spectral index is $-0.87 \pm 0.01$, calculated using the Kaplan-Meier estimator (KM; e.g. Feigelson \& Nelson 1985) to account for the upper limits. This value is comparable to those found by Intema et al. (2011), -0.79, Ishwara-Chandra \& Marathe (2007), -0.85, Sirothia et al. (2009), -0.82, and Ishwara-Chandra et al. (2010), -0.78. By considering the KM mean spectral index

\footnotetext{
4 The spectral index is defined as $S_{v} \propto v^{\alpha}$.
}

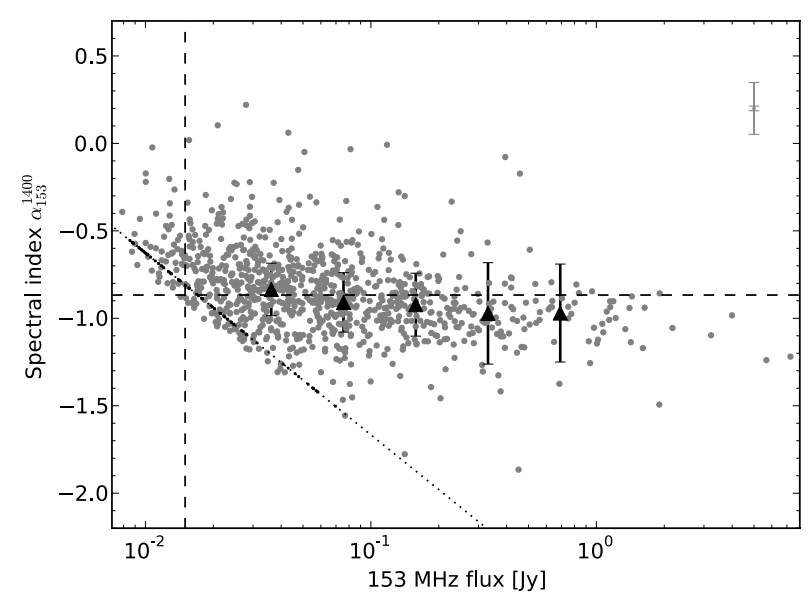

Fig. 9. Spectral index, $\alpha_{153}^{1400}$, distribution of sources matched between $1.4 \mathrm{GHz}$ and $153 \mathrm{MHz}$ (grey points). The difference in resolution is 45" (NVSS) and 25" (GMRT) and multiple GMRT matches to a single NVSS source have been merged into one. The vertical line shows $5 \sigma_{\text {avg }}$, where $\sigma_{\text {avg }}$ is the average rms noise in the GMRT mosaic. The diagonal dotted line indicates the incompleteness limit due to the sensitivity of NVSS and sources with upper limits are plotted as black points along this line. The horizontal dashed line shows the KM mean spectral index of $-0.87 \pm 0.01$ accounting for upper limits. The large black triangles show the mean spectral index in 5 logarithmic bins. Error bars on individual points are not plotted for clarity, but a single bar in the top right indicates the maximum and minumum errors in the dataset.

within 5 logarithmic flux density bins between $85 \mathrm{mJy}$ and $1 \mathrm{Jy}$ (overplotted in Fig. 9 and listed in Table 6), we find a gradual steepening of the spectral index with increasing flux density, from $\sim-0.84$ at $\sim 30 \mathrm{mJy}$ to $\sim-0.97$ at $F \gtrsim 600 \mathrm{mJy}$. This trend is still clear if the first flux density bin is ignored (i.e. considering $F \gtrsim 40 \mathrm{mJy}$ ) assuming that this bin remains biased by the upper limits. This is consistent with what is found in the literature (e.g. Ishwara-Chandra et al. 2010; Tasse et al. 2006; Cohen et al. 2004; de Vries et al. 2002). Since it appears that there is no 
W. L. Williams et al.: T-RaMiSu: The Two-meter Radio Mini Survey. I.

Table 6. Binned median spectral indices between the GMRT at $153 \mathrm{MHz}$ and NVSS at $1.4 \mathrm{GHz}$ and WENSS at $327 \mathrm{MHz}$ ).

\begin{tabular}{lcccc}
\hline \hline $\begin{array}{c}\text { Bin centre } \\
{[\mathrm{mJy}]}\end{array}$ & $\begin{array}{c}\text { Counts } \\
\text { (Upper limits) }\end{array}$ & $\begin{array}{c}\text { NVSS } \alpha_{153}^{1400} \\
\text { KM mean spectral index }\end{array}$ & $\begin{array}{c}\text { Counts } \\
\text { (Upper limits) }\end{array}$ & $\begin{array}{c}\text { WENSS } \alpha_{153}^{327} \\
\text { KM mean spectral index }\end{array}$ \\
\hline 36 & $275(10)$ & $-0.835 \pm 0.015$ & - & - \\
76 & $213(7)$ & $-0.909 \pm 0.017$ & $209(16)$ & $-0.940 \pm 0.031$ \\
158 & 143 & $-0.922 \pm 0.018$ & 140 & $-0.752 \pm 0.028$ \\
331 & 71 & $-0.972 \pm 0.029$ & 72 & $-0.792 \pm 0.028$ \\
692 & 28 & $-0.970 \pm 0.028$ & 32 & $-0.845 \pm 0.033$ \\
\hline
\end{tabular}

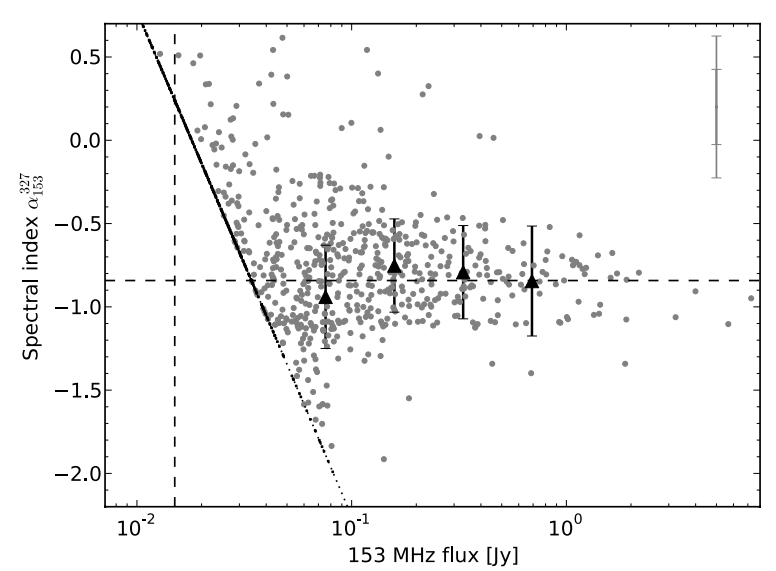

Fig. 10. Spectral index, $\alpha_{153}^{327}$, distribution between $327 \mathrm{MHz}$ and $153 \mathrm{MHz}$ (grey points). The difference in resolution is $54^{\prime \prime} \times 96^{\prime \prime}$ (WENSS) and 25" (GMRT) and multiple GMRT matches to a single WENSS source have been merged. The vertical line shows $5 \sigma_{\text {avg }}$, where $\sigma_{\text {avg }}$ is the average rms noise in the GMRT mosaic. The diagonal dotted line indicates the incompleteness limit due to the sensitivity of WENSS. The horizontal dashed line shows the KM mean spectral index of $-0.84 \pm 0.02$ which takes the upper limits into account. The large black triangles show the median spectral index in 4 logarithmic bins. Error bars on individual points are not plotted for clarity, but a single bar in the top right indicates the maximum and minumum errors in the dataset.

spectral steepening or flattening due to redshifted curved spectra (Bornancini et al. 2010), this flattening is likely due to a correlation between source luminosity and spectral index $(P-\alpha)$, which is known to exist for FRII radio galaxies (e.g. Blundell et al. 1999). According to the models of Wilman et al. (2008), the observed $153 \mathrm{MHz}$ source population is dominated by FRII galaxies at these flux density levels ( $\gtrsim 20 \mathrm{mJy}$ ).

We also compared our source list to WENSS at $327 \mathrm{MHz}$ (Rengelink et al. 1997), noting that the errors in this spectral index are much greater due to the smaller difference in frequency. The WENSS beam is $54^{\prime \prime} \times 54^{\prime \prime} / \sin \delta$, or $54^{\prime \prime} \times 96^{\prime \prime}$ at the declination of the Boötes field. We thus searched for WENSS counterparts within $96^{\prime \prime}$ of each GMRT source. Of the 1289 GMRT sources we matched 689 to 675 WENSS sources. The 14 pairs of GMRT sources within the beam of a single WENSS source were combined as described in the previous paragraph and spectral indices determined for each based on the combined flux density. A visual check led to the removal of 12 misidentified or confused sources. The resulting spectral index distribution for $\alpha_{153}^{327}$ is shown in Fig. 10. Once again there is a bias towards flatter or inverted spectra at low $153 \mathrm{MHz}$ flux densities due to the WENSS flux density limit of $18 \mathrm{mJy}$ at $327 \mathrm{MHz}$. We provide upper limits to the spectral indices given the WENSS flux density limit for the 576 GMRT sources with that have WENSS flux densities below the WENSS detection limit and thus should have spectral indices are steeper

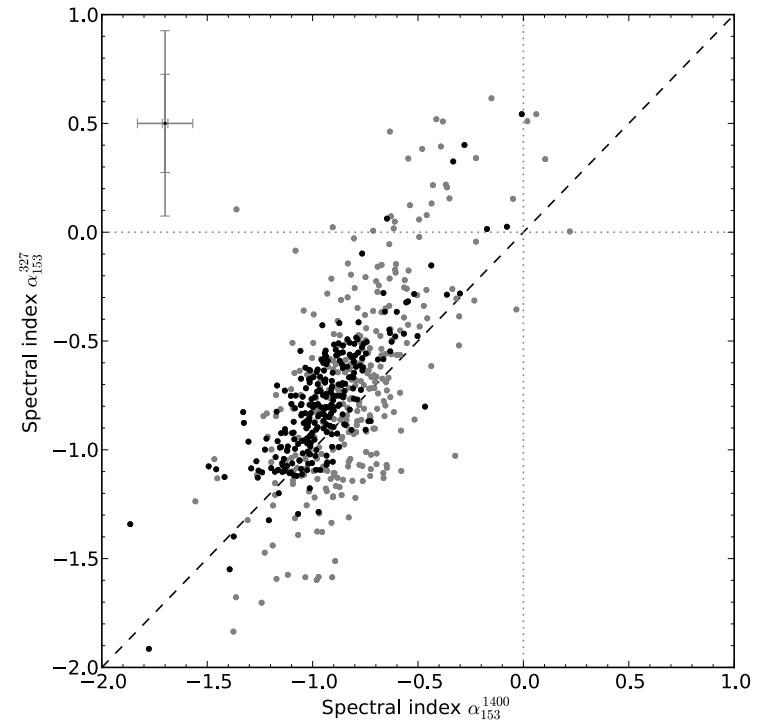

Fig. 11. Comparison between $\alpha_{327}^{1400}$ and $\alpha_{153}^{327}$. The black dashed line indicates where the spectral index is the same in both regions of the spectrum. Sources with GMRT fluxes above $0.1 \mathrm{Jy}^{\text {beam }}{ }^{-1}$ are plotted in black and fainter sources are plotted in grey. Error bars on individual points are not plotted for clarity, but a single error bar in the top left indicates the maximum and minumum errors in the dataset.

than the diagonal line in Fig. 10. The KM mean spectral index in this case is $-0.84 \pm 0.02$ (taking the upper limits into account) and is slightly shallower than that observed between 153 and $1400 \mathrm{MHz}$. The KM mean spectral indices measured in 4 flux density bins are also listed in Table 6. There is, however, no clear trend with flux density observed, although there is an indication of a slight flattening of the average radio spectrum if the first flux bin is excluded. This may be due to the fact that $\alpha_{153}^{327}$ is less robust due to the small frequency difference and the errors on the individual measurements are higher.

Around 50 per cent of our sources have data at three frequencies $(1400,327$, and $153 \mathrm{MHz})$, thus we have not attempted to fit or locate peaks in the radio spectra. Instead we show a radio "colour-colour" plot, Fig. 11, comparing the spectral indices $\alpha_{153}^{1400}$ and $\alpha_{153}^{327}$. The line illustrates where the two spectral indices are equal. Here we have plotted separately bright $153 \mathrm{MHz}$ sources, above $0.1 \mathrm{Jy}$ beam $^{-1}$, as these sources have smaller errors on their spectral indices and are not affected by incompleteness at the other two frequencies (see Figs. 9 and 10). In general there is a flattening of the average radio spectrum towards lower frequencies, as the majority of points fall above the line. It is likely that this observed turnover in the spectra at low frequencies is due synchrotron self-absorption. We also plot the distribution of the difference in spectral indices, $\alpha_{153}^{1400}-\alpha_{153}^{327}$, Fig. 12, which shows a mean value of -0.25 for only bright sources and and -0.2 for all sources. 


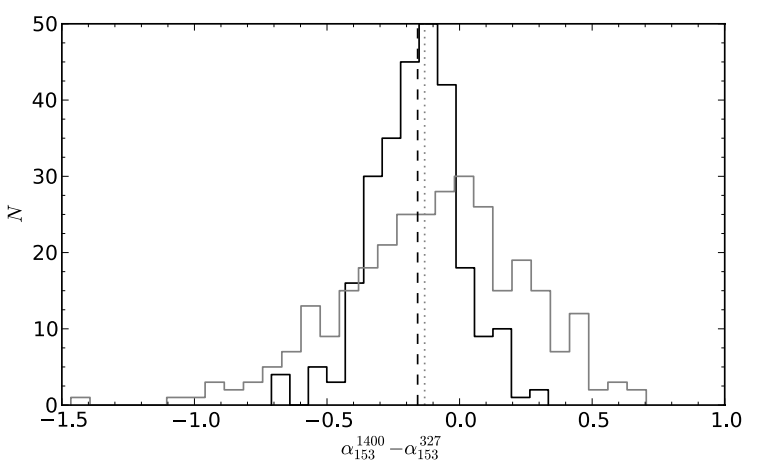

Fig. 12. Comparison between $\alpha_{153}^{1400}$ and $\alpha_{153}^{327}$ : histogram of $\alpha_{153}^{1400}-\alpha_{153}^{327}$. Again, the histogram for bright GMRT sources is plotted in black and for fainter sources in grey. The dashed black line shows the mean value of -0.25 and the grey dotted line, the mean value of -0.2 , for bright and all sources respectively, indicating that the majority of sources have flattened spectra at low frequencies.

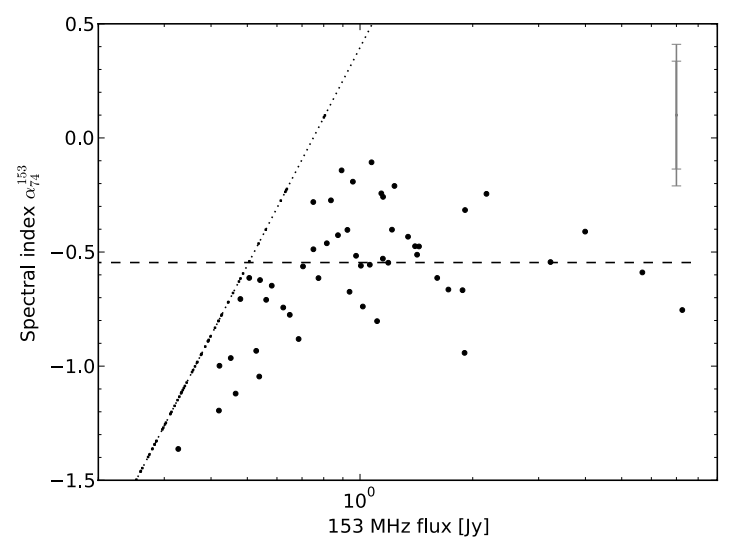

Fig. 13. Spectral index distribution between $74 \mathrm{MHz}$ and $153 \mathrm{MHz}$. The difference in resolution is $80^{\prime \prime}$ (VLSS) and the 25" (GMRT) and multiple GMRT matches to a single VLSS source have been merged into one. The vertical line shows $5 \sigma_{\text {avg }}$, where $\sigma_{\text {avg }}$ is the average rms noise in the GMRT mosaic. The diagonal dotted line indicates the incompleteness limit due to the sensitivity of VLSS. The horizontal dashed line shows the KM mean spectral index of -0.55 . Error bars on individual points are not plotted for clarity, but a single bar in the top right indicates the maximum and minumum errors in the dataset.

Finally, we have also compared our source list to VLSS at $74 \mathrm{MHz}$ (Cohen et al. 2007), again noting that the errors in this spectral index will be much greater due to the smaller difference in frequency. VLSS has a resolution of $80^{\prime \prime}$ so we searched for VLSS sources within this radius of each GMRT source. 58 GMRT sources were matched to 55 VLSS sources. The resulting spectral index distribution is shown in Fig. 13. In this case there is a bias towards steeper spectra at low $153 \mathrm{MHz}$ flux densities due to the VLSS flux density limit of $0.5 \mathrm{Jy}$ at $74 \mathrm{MHz}$. The KM mean spectral index in this case is -0.55 which was calculated for sources with GMRT fluxes above $0.5 \mathrm{Jy}$.

\section{Conclusion}

We have presented the results from a $\sim 30$ square degree, high resolution $\left(25^{\prime \prime}\right)$ radio survey at $153 \mathrm{MHz}$ centred on the NOAO Boötes field. We have employed the SPAM ionospheric calibration scheme to achieve an rms noise in the 7 pointing mosaicked image of $\sim 2-4 \mathrm{mJybeam}^{-1}$. The source catalogue contains 1289 sources between $4.1 \mathrm{mJy}$ and $7.3 \mathrm{Jy}$ detected at 5 times the local noise. We estimate the catalogue to be 92 per cent reliable and 95 per cent complete to an integrated
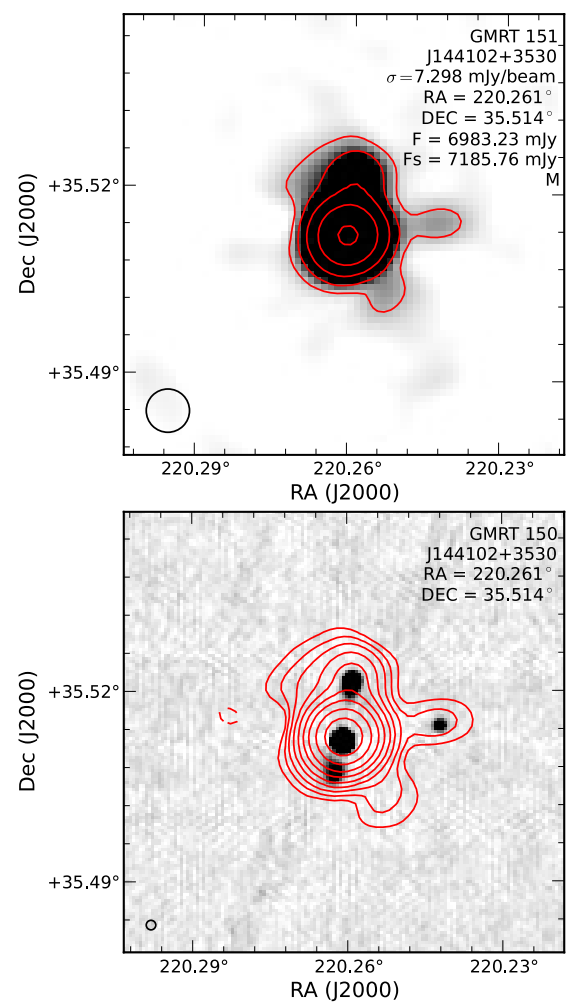

Fig. A.1. The second brightest source in the $153 \mathrm{MHz}$ catalogue: (top) GMRT image and (bottom) FIRST image with GMRT contours. In both images the GMRT contours are plotted in red at intervals of $3 \sigma \times[-\sqrt{3}, \sqrt{3}, \sqrt{10}, \sqrt{30}, \sqrt{100}, \ldots]$ and the greyscale goes from $1 \sigma$ to $30 \sigma$.

flux density of $14 \mathrm{mJy}$. The catalogue has been corrected for systematic errors on both the astrometry and flux density scales.

We have analysed the source population by investigating the source counts and by identifying counterparts within the $1.4 \mathrm{GHz}$ NVSS and $327 \mathrm{MHz}$ WENSS surveys and have computed the spectral index distributions of these sources. Understanding the low frequency, low flux source population is of particular importance to Epoch of Reionization projects (e.g. Ghosh et al. 2012, and references therein) where good models of the foregrounds are needed.

In the near future, this data will be combined with the existing multi-wavelength data covering the NOAO Boötes field and we will study the properties of radio galaxies as a function of various multi-wavelength parameters across a range of cosmic time. Further investigation of the spectral indices will be done and can be used to identify USS sources as well as high redshift gigahertz peaked spectrum (GPS) sources.

Acknowledgements. The authors thank the anonymous referee for useful comments, which have improved this manuscript. We also acknowledge the staff of the GMRT that made these observations possible. GMRT is run by the National Centre for Radio Astrophysics of the Tata Institute of Fundamental Research. This publication made use of data from the Very Large Array, operated by the National Radio astronomy Observatory. The National Radio Astronomy Observatory is a facility of the National Science Foundation operated under cooperative agreement by Associated Universities, Inc.

\section{Appendix A: Selected radio images}

Figures A.2 shows the 25 brightest sources in the catalogue, excluding the second brightest source which is described below (see also Fig. A.1). 
W. L. Williams et al.: T-RaMiSu: The Two-meter Radio Mini Survey. I.
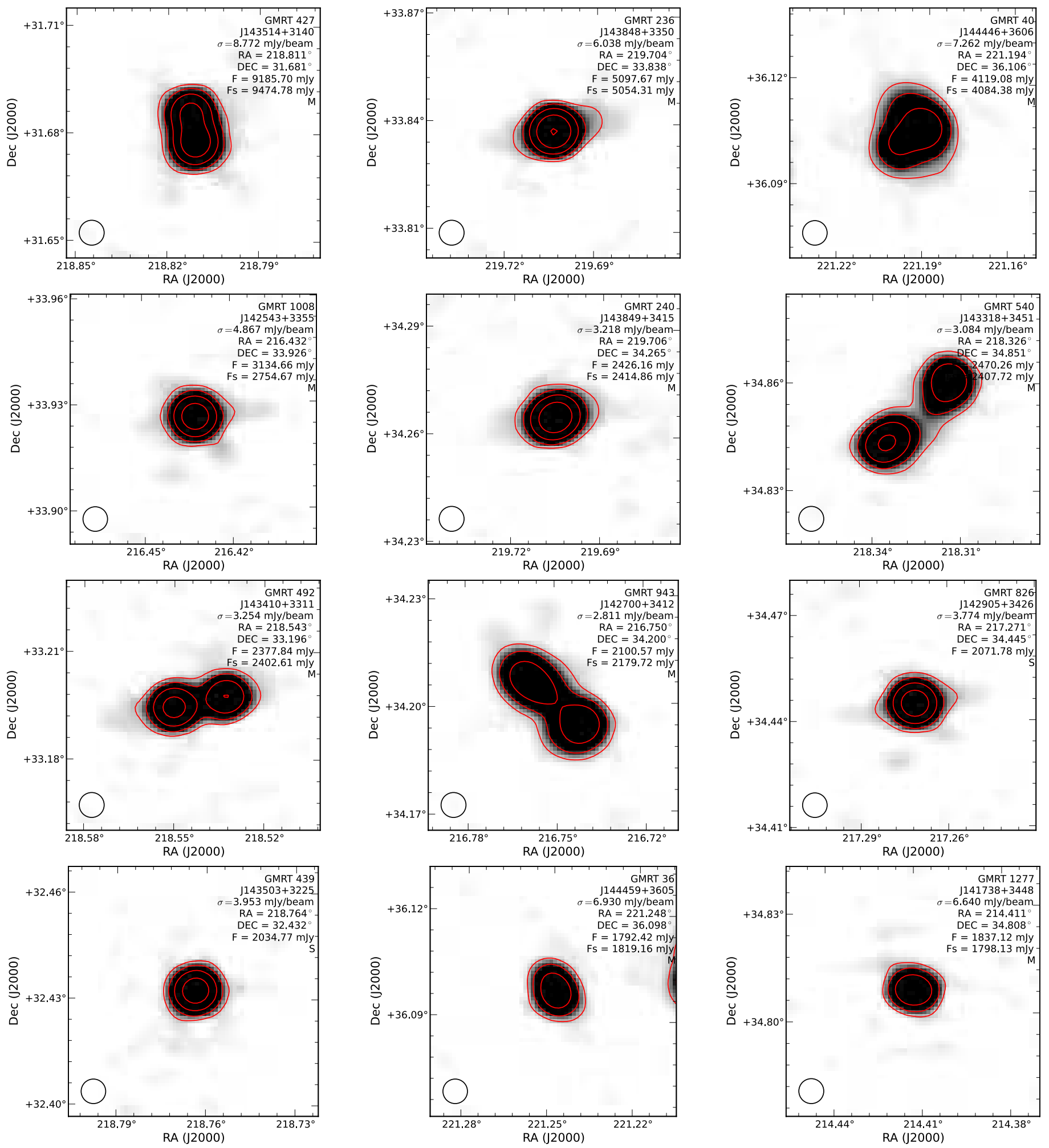

Fig. A.2. The 25 brightest $153 \mathrm{MHz}$ radio sources (exluding J144102+3530). Contours are plotted in red at intervals of $3 \sigma \times$ $(-\sqrt{3}, \sqrt{3}, \sqrt{10}, \sqrt{30}, \sqrt{100}, \ldots)$ and the greyscale goes from $1 \sigma$ to $30 \sigma$. The text in each image lists the local rms noise, the source coordinates and total flux, density and the source type ("S" or "M"). The beamsize is shown in the bottom left corner.

\section{A.1. Note on source J144102+3530}

Figure A.1 shows GMRT postage stamp of the second brightest source in the catalogue. Also shown is the FIRST image
(Becker et al. 1995) of this source which shows that most of the structure seen in the GMRT image is in fact real. Only the extension to the North-West in the GMRT image has no clear match in the FIRST image and may be due to deconvolution errors. 

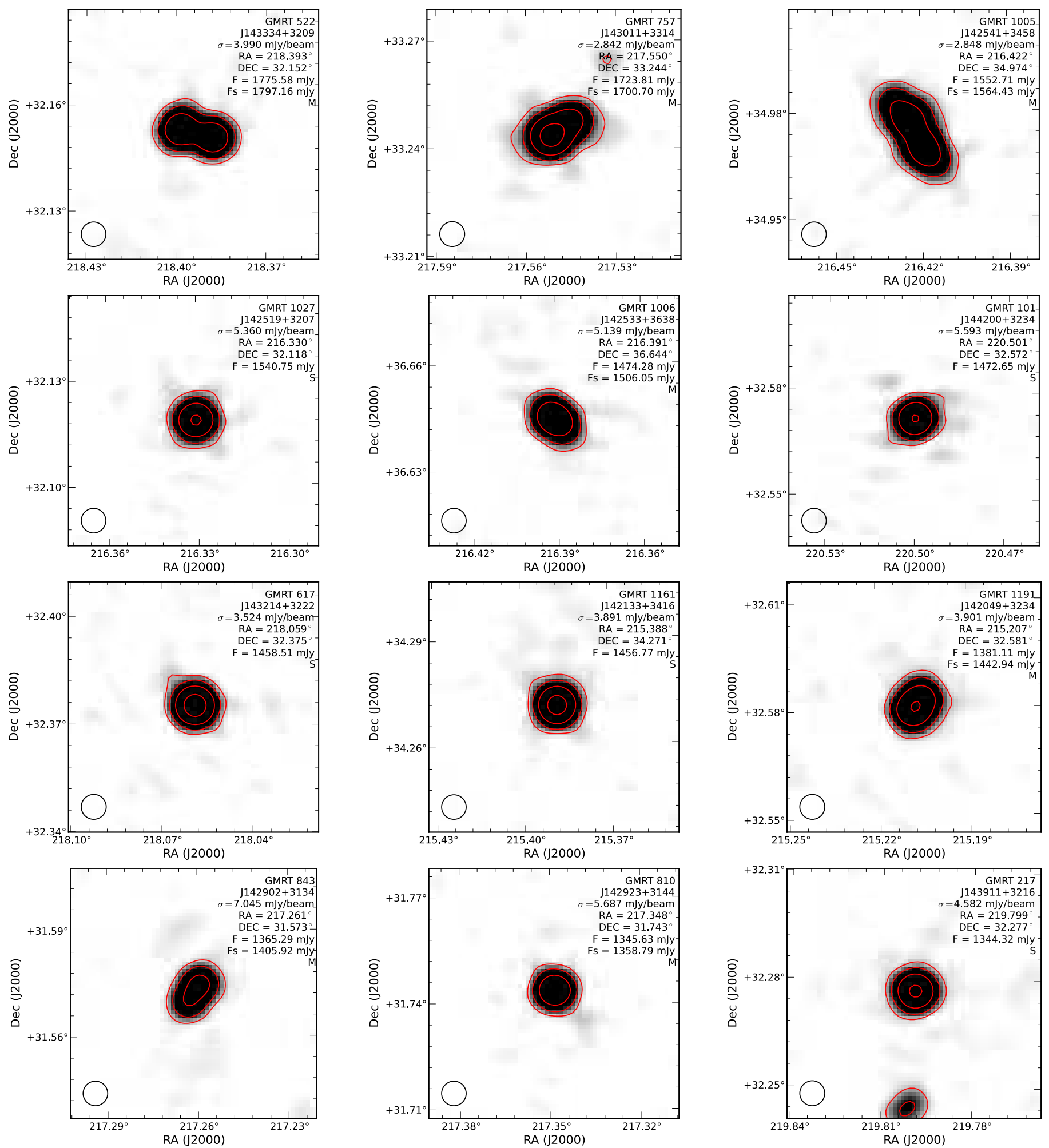

Fig. A.2. continued.

\section{References}

Ananthakrishnan, S. 2005, in International Cosmic Ray Conference, 10, 125 Becker, R. H., White, R. L., \& Helfand, D. J. 1995, ApJ, 450, 559

Best, P. N., Kauffmann, G., Heckman, T. M., et al. 2005, MNRAS, 362, 25

Best, P. N., Kaiser, C. R., Heckman, T. M., \& Kauffmann, G. 2006, MNRAS, 368, L67

Best, P. N., von der Linden, A., Kauffmann, G., Heckman, T. M., \& Kaiser, C. R. 2007, MNRAS, 379, 894
Blundell, K. M., Rawlings, S., \& Willott, C. J. 1999, AJ, 117, 677

Bornancini, C. G., O’Mill, A. L., Gurovich, S., \& Lambas, D. G. 2010, MNRAS 406, 197

Bower, R. G., Benson, A. J., Malbon, R., et al. 2006, MNRAS, 370, 645

Briggs, D. S. 1995, in BAAS, 27, AAS Meeting Abstracts, 112.02

Chandra, P., Ray, A., \& Bhatnagar, S. 2004, ApJ, 612, 974

Clemens, M. S., Scaife, A., Vega, O., \& Bressan, A. 2010, MNRAS, 405, 887

Cohen, A. S., Röttgering, H. J. A., Jarvis, M. J., Kassim, N. E., \& Lazio, T. J. W. 2004, ApJS, 150, 417 
W. L. Williams et al.: T-RaMiSu: The Two-meter Radio Mini Survey. I.

Cohen, A. S., Lane, W. M., Cotton, W. D., et al. 2007, AJ, 134, 1245

Condon, J. J. 1997, PASP, 109, 166

Condon, J. J., Cotton, W. D., Greisen, E. W., et al. 1994, in Astronomical Data Analysis Software and Systems III, eds. D. R. Crabtree, R. J. Hanisch, \& J. Barnes, ASP Conf. Ser., 61, 155

Condon, J. J., Cotton, W. D., Greisen, E. W., et al. 1998, AJ, 115, 1693

Conway, J. E., Cornwell, T. J., \& Wilkinson, P. N. 1990, MNRAS, 246, 490

Cornwell, T. J., \& Perley, R. A. 1992, A\&A, 261, 353

Cornwell, T., Braun, R., \& Briggs, D. S. 1999, in Synthesis Imaging in Radio Astronomy II, eds. G. B. Taylor, C. L. Carilli, \& R. A. Perley, ASP Conf. Ser., 180,151

Cotton, W. D. 1999, in Synthesis Imaging in Radio Astronomy II, eds. G. B. Taylor, C. L. Carilli, \& R. A. Perley, ASP Conf. Ser., 180, 357

Cotton, W. D. 2008, in PASP, 120, 439

Cotton, W. D., Condon, J. J., Perley, R. A., et al. 2004, in SPIE Conf. Ser. 5489, ed. J. M. Oschmann, Jr., 180

Croft, S., van Breugel, W., Brown, M. J. I., et al. 2008, AJ, 135, 1793

Croton, D. J., Springel, V., White, S. D. M., et al. 2006, MNRAS, 365, 11

De Breuck, C., van Breugel, W., Stanford, S. A., et al. 2002, AJ, 123, 637

de Vries, W. H., Morganti, R., Röttgering, H. J. A., et al. 2002, AJ, 123, 1784

Delain, K. M., \& Rudnick, L. 2006, Astron. Nachr., 327, 561

Eisenhardt, P. R., Stern, D., Brodwin, M., et al. 2004, ApJS, 154, 48

Fabian, A. C., Celotti, A., \& Erlund, M. C. 2006, MNRAS, 373, L16

Feigelson, E. D., \& Nelson, P. I. 1985, ApJ, 293, 192

Gehrels, N. 1986, ApJ, 303, 336

George, S. J., \& Stevens, I. R. 2008, MNRAS, 390, 741

Ghosh, A., Prasad, J., Bharadwaj, S., Saiyad Ali, S., \& Chengalur, J. N. 2012, MNRAS, 426, 3295

Greisen, E. W. 1998, in Astronomical Data Analysis Software and Systems VII, eds. R. Albrecht, R. N. Hook, \& H. A. Bushouse, ASP Conf. Ser., 145, 204

Haslam, C. G. T., Salter, C. J., Stoffel, H., \& Wilson, W. E. 1982, A\&AS, 47, 1 Higdon, J. L., Higdon, S. J. U., Weedman, D. W., et al. 2005, ApJ, 626, 58

Intema, H. T., van der Tol, S., Cotton, W. D., et al. 2009, A\&A, 501, 1185

Intema, H. T., van Weeren, R. J., Röttgering, H. J. A., \& Lal, D. V. 2011, A\&A, 535, A 38
Ishwara-Chandra, C. H., \& Marathe, R. 2007, in Deepest Astronomical Surveys, eds. J. Afonso, H. C. Ferguson, B. Mobasher, \& R. Norris, ASP Conf. Ser., 380,237

Ishwara-Chandra, C. H., Sirothia, S. K., Wadadekar, Y., Pal, S., \& Windhorst, R. 2010, MNRAS, 405, 436

Jannuzi, B. T., Dey, A., \& NDWFS Team 1999, in, AAS Meeting Abstracts, BAAS31, 1392

Kenter, A., Murray, S. S., Forman, W. R., et al. 2005, ApJS, 161, 9

Kettenis, M., van Langevelde, H. J., Reynolds, C., \& Cotton, B. 2006, in Astronomical Data Analysis Software and Systems XV, eds. C. Gabriel, C. Arviset, D. Ponz, \& S. Enrique, PASPC, 351, 497

Kochanek, C. S., Eisenstein, D. J., Cool, R. J., et al. 2012, ApJS, 200, 8

Kovács, A., Chapman, S. C., Dowell, C. D., et al. 2006, ApJ, 650, 592

Martin, C., Barlow, T., Barnhart, W., et al. 2003, in SPIE Conf. Ser. 4854, eds. J. C. Blades, \& O. H. W. Siegmund, 336

McGilchrist, M. M., Baldwin, J. E., Riley, J. M., et al. 1990, MNRAS, 246, 110

Miley, G., \& De Breuck, C. 2008, A\&ARv, 15, 67

Mohan, R., Dwarakanath, K. S., Srinivasan, G., \& Chengalur, J. N. 2001, J. Astrophys. Astron., 22, 35

Murray, S. S., Kenter, A., Forman, W. R., et al. 2005, ApJS, 161, 1

Perley, R. A. 1989, in Synthesis Imaging in Radio Astronomy, eds. R. A. Perley, F. R. Schwab, \& A. H. Bridle, ASP Conf. Ser., 6, 287

Polatidis, A. G., \& Conway, J. E. 2003, PASA, 20, 69

Rengelink, R. B., Tang, Y., de Bruyn, A. G., et al. 1997, A\&AS, 124, 259

Röttgering, H. J. A., van Ojik, R., Miley, G. K., et al. 1997, A\&A, 326, 505

Schwab, F. R. 1984, AJ, 89, 1076

Sirothia, S. K., Saikia, D. J., Ishwara-Chandra, C. H., \& Kantharia, N. G. 2009, MNRAS, 392, 1403

Snellen, I. A. G., Mack, K.-H., Schilizzi, R. T., \& Tschager, W. 2003, PASA, 20, 38

Tasse, C., Cohen, A. S., Röttgering, H. J. A., et al. 2006, A\&A, 456, 791

Tasse, C., Röttgering, H. J. A., Best, P. N., et al. 2007, A\&A, 471, 1105

Wilman, R. J., Miller, L., Jarvis, M. J., et al. 2008, MNRAS, 388, 1335

Windhorst, R. A., Miley, G. K., Owen, F. N., Kron, R. G., \& Koo, D. C. 1985 , ApJ, 289, 494 